\title{
Galactic-scale Feedback Observed in the 3C 298 Quasar Host Galaxy
}

\author{
Andrey Vayner $^{1,2}$, Shelley A. Wright ${ }^{1,2}$, Norman Murray ${ }^{3,4}$, Lee Armus ${ }^{5}$, James E. Larkin ${ }^{6}$ (1), and Etsuko Mieda ${ }^{7}$ \\ ${ }_{1}^{1}$ Department of Physics, University of California San Diego, 9500 Gilman Drive La Jolla, CA 92093, USA; avayner@ucsd.edu \\ ${ }_{3}^{2}$ Center for Astrophysics \& Space Sciences, University of California San Diego, 9500 Gilman Drive La Jolla, CA 92093, USA \\ ${ }^{3}$ Canadian Institute for Theoretical Astrophysics, University of Toronto, 60 St. George Street, Toronto, ON M5S 3H8, Canada \\ ${ }^{4}$ Canada Research Chair in Theoretical Astrophysics \\ ${ }^{5}$ Spitzer Science Center, California Institute of Technology, 1200 E. California Boulevard, Pasadena, CA 91125, USA \\ ${ }^{6}$ Department of Physics and Astronomy, University of California, Los Angeles, CA 90095, USA \\ ${ }^{7}$ NRC Herzberg Astronomy and Astrophysics, 5071 West Saanich Road, Victoria, BC V9E 2E7, Canada \\ Received 2017 September 11; revised 2017 November 3; accepted 2017 November 15; published 2017 December 20
}

\begin{abstract}
We present high angular resolution multiwavelength data of the 3C 298 radio-loud quasar host galaxy $(z=1.439)$ taken using the W.M. Keck Observatory OSIRIS integral field spectrograph (IFS) with adaptive optics, the Atacama Large Millimeter/submillimeter Array (ALMA), the Hubble Space Telescope (HST) WFC3, and the Very Large Array (VLA). Extended emission is detected in the rest-frame optical nebular emission lines $\mathrm{H} \beta$, [O III], $\mathrm{H} \alpha$, [N II] and [S II], as well as in the molecular lines $\mathrm{CO}(J=3-2)$ and $(J=5-4)$. Along the path of the relativistic jets of 3C 298, we detect conical outflows in ionized gas emission with velocities of up to $1700 \mathrm{~km} \mathrm{~s}^{-1}$ and an outflow rate of $450-1500 M_{\odot} \mathrm{yr}^{-1}$ extended over $12 \mathrm{kpc}$. Near the spatial center of the conical outflow, $\mathrm{CO}$ $(J=3-2)$ emission shows a molecular gas disk with a rotational velocity of $\pm 150 \mathrm{~km} \mathrm{~s}^{-1}$ and total molecular mass $\left(M_{\mathrm{H}_{2}}\right)$ of $6.6 \pm 0.36 \times 10^{9} M_{\odot}$. On the blueshifted side of the molecular disk, we observe broad extended emission that is due to a molecular outflow with a rate of $2300 M_{\odot} \mathrm{yr}^{-1}$ and depletion timescale of $3 \mathrm{Myr}$. We detect no narrow $\mathrm{H} \alpha$ emission in the outflow regions, suggesting a limit on star formation of $0.3 M_{\odot} \mathrm{yr}^{-1} \mathrm{kpc}^{-2}$. Quasardriven winds are evacuating the molecular gas reservoir, thereby directly impacting star formation in the host galaxy. The observed mass of the supermassive black hole is $10^{9.37-9.56} M_{\odot}$, and we determine a dynamical bulge mass of $M_{\text {bulge }}=1-1.7 \times 10^{10} \frac{R}{1.6 \mathrm{kpc}} M_{\odot}$. The bulge mass of 3C 298 lies 2-2.5 orders of magnitude below the expected value from the local galactic bulge-supermassive black hole mass $\left(M_{\text {bulge }}-M_{\mathrm{BH}}\right)$ relationship. A second galactic disk observed in nebular emission is offset from the quasar by $9 \mathrm{kpc}$, suggesting that the system is an intermediate-stage merger. These results show that galactic-scale negative feedback is occurring early in the merger phase of 3C 298, well before the coalescence of the galactic nuclei and assembly on the local $M_{\mathrm{bulge}}-M_{\mathrm{BH}}$ relationship.
\end{abstract}

Key words: galaxies: active - galaxies: high-redshift - galaxies: kinematics and dynamics - quasars: emission lines - quasars: individual (3C 298) - quasars: supermassive black holes

\section{Introduction}

Quasars are the most luminous active galactic nuclei (AGN), whose supermassive black holes (SMBHs) are often fueled by large galaxy mergers (Treister et al. 2012). AGN accretion disks are thought to drive energetic winds (Murray et al. 1995) and/or relativistic jets that suppress star formation (Hopkins \& Elvis 2010; Zubovas \& King 2014; Costa et al. 2015), thereby impacting galactic structure and evolution. This is one of the leading theoretical (Di Matteo et al. 2005; Faucher-Giguère et al. 2012; Barai et al. 2017; Anglés-Alcázar et al. 2017) pictures used to explain correlations between SMBH masses and galactic stellar masses (Magorrian et al. 1998; Ferrarese \& Merritt 2000; Gebhardt et al. 2000; Kormendy \& Ho 2013) and the luminosity function of local massive galaxies (Benson et al. 2003). The sphere of influence of SMBHs, inside which their gravity dominates that of the stars, gas, and dark matter, range from a few $10 \mathrm{~s}$ to $100 \mathrm{pc}$, while the local scaling relations of $M_{\text {bulge }}-M_{\mathrm{BH}}$ apply on stellar bulge scales, i.e., several kpc. The SMBH energy output can be orders of magnitude higher than the binding energy of galactic bulges; therefore energy injected by active SMBHs into the interstellar medium (ISM) could be efficient at impacting the stellar mass history of its host galaxy. Since the bulk of stellar mass is formed at high redshift $(z>1$; Gallazzi et al. 2008), it is critical to study the effects of AGN activity during the assembly periods of their host galaxies at high redshift.

Observational studies have found that nearby quasars and ultra-luminous infrared galaxies (ULIRGs) show evidence of large-scale ionized (Rupke \& Veilleux 2011; Greene et al. 2012; Liu et al. 2013a; Harrison et al. 2014) and molecular outflows (e.g., Cicone et al. 2014; Sun et al. 2014; Stone et al. 2016; Veilleux et al. 2017), allowing for detailed studies of feedback physics. However, the computed outflow rates in nearby systems are not sufficient to impact the stellar mass assembly history since these galaxies have completed the bulk of their growth. In contrast, there is minimal observational evidence for AGN activity directly affecting star formation at the peak epoch $(1<z<3)$ of galaxy formation and black hole accretion (Delvecchio et al. 2014; Madau \& Dickinson 2014). Recent studies of distant quasars and radio-loud galaxies have shown evidence of large-scale $(5-20 \mathrm{kpc})$ outflows driven by AGN activity that theoretically should be powerful enough to quench star formation (Nesvadba et al. 2008; Steinbring 2011; Cano-Díaz et al. 2012; Harrison et al. 2012; Carniani et al. 2015; Brusa et al. 2016), although it has still remained challenging to directly associate large-scale outflows with the suppression of star formation of AGN host galaxies (Cano-Díaz et al. 2012; Cresci et al. 2015; Carniani et al. 2016). These studies have provided intriguing clues as to the nature of quasar 
feedback. Compared to nearby quasars, however, there is little known about $z>1$ host galaxies (i.e., stellar mass, dynamics, and morphologies) and the true effects of feedback on host galaxy star formation. A key missing result is one that connects quasar and jet-driven outflows with the observed galactic-scale winds, star formation activity, and molecular gas properties.

Quasars outshine their host galaxies by an order of magnitude or more, making it observationally challenging to detect and study their faint underlying galaxies (Lehnert et al. 1999; Hutchings et al. 2002; Jahnke et al. 2004; Falomo et al. 2005; Floyd et al. 2013; Glikman et al. 2015). The sizes of distant $(z>1$; look-back time of $9.25 \mathrm{Gyr})$ galaxies are small $\left(\sim 1^{\prime \prime}\right)$, roughly the same angular size as ground-based "seeing" and space-based instrument resolution and contrast sensitivity. The combination of near-infrared integral field spectroscopy (IFS) with laser guide star adaptive optics (LGSAO) allows for the effective separation of quasar emission from the host galaxy. This is achieved using a pristine point-spread function (PSF) generated by the quasar broad-line and continuum emission from the IFS data cube (Inskip et al. 2011; Vayner et al. 2016).

We have started the survey called Quasar hosts Unveiled by high Angular Resolution Techniques (QUART) of highredshift $(1.3<z<2.6)$ quasars using W.M Keck Observatory LGS-AO observations to resolve and study quasar host galaxy properties with rich multiwavelength data sets. Herein we present the first paper of this survey on the individual $z=1.439$ (look-back time $9.3 \mathrm{Gyr}$ ) radio-loud quasar 3C 298. We combine high spatial resolution observations to reveal the complex morphology, dynamics, and energetics of the 3C 298 host galaxy. Using OSIRIS and Keck LGS-AO, we are able to map the kinematics and intensity of the galactic nebular emission lines $\mathrm{H} \beta,[\mathrm{O} \mathrm{III]}, \mathrm{H} \alpha,[\mathrm{N} \mathrm{II}]$, and [S II] at $~ 1.4$ kiloparsec $(\mathrm{kpc})$ resolution. We present the multiwavelength data sets in Section 2 and describe the data reduction and analysis techniques for each instrument in Section 3. We discuss the dynamics and energetics of ionized and molecular gas in Section 4, and in Section 5 we discuss our results and overall interpretation. We suggest the reader refers to Figure 11 while reading the manuscript, which summarizes the observed structure and properties of the 3C 298 host galaxy. Throughout the paper we assume a $\Lambda$-dominated cosmology (Planck Collaboration et al. 2014) with $\Omega_{M}=0.308, \Omega_{\Lambda}=0.692$, and $\mathrm{H}_{o}=67.8 \mathrm{~km} \mathrm{~s}^{-1} \mathrm{Mpc}^{-1}$.

\section{Observations}

We present new observations of 3C 298 using Keck OSIRIS AO and ALMA band 4 and 6. These observations are coupled with archival Hubble Space Telescope (HST) WFC3 and Very Large Array (VLA) imaging data.

\subsection{Keck: OSIRIS}

Observations were taken using the integral field spectrograph OSIRIS (Larkin et al. 2006) with the upgraded grating (Mieda et al. 2014) behind the LGS-AO system at W. M. Keck Observatory on 2014 May 19 and 20, (UT). The quasar was used for tip/tilt correction, while the laser tuned to $589.2 \mathrm{~nm}$ created an artificial star on-axis for higher order corrections. We used the Hn3 (May 19) and Jn1 (May 20) filters with a plate scale of 100 milliarcseconds (mas) per lenslet with a position angle of $103^{\circ}$. In these modes, OSIRIS has a field of view of 3 !" $2 \times 6$ " 4 in Jn1 and 4 " $8 \times 6$ " 4 in $\mathrm{Hn} 3$. We took four 600s exposures on-source in each filter, plus an additional 600s pure-sky frame. Each night immediately after the quasar observations, we observed the standard star HD136754 for telluric and flux calibrations. Both nights were photometric with near-infrared seeing of 0 ". $4-0$ ". 5 .

\subsection{ALMA: Bands 4 and 6}

Early ALMA science (cycles 2 and 3) band 4 and band 6 observations (2013.1.01359.S and 2015.1.01090.S, PI: Vayner) were aimed at observing the rotational molecular transition of CO $J=3-2$ and $J=5-4$ in emission to map the distribution and kinematics of the molecular gas in 3C 298. One $1.8745 \mathrm{GHz}$ spectral window was centered on $\mathrm{CO}(J=3-2)$ $(141.87 \mathrm{GHz})$ and $\mathrm{CO}(J=5-4)(236.43 \mathrm{GHz})$, while three additional spectral windows were set up to map the continuum in each band. The effective velocity bandwidth per spectral window was approximately $4000 \mathrm{~km} \mathrm{~s}^{-1}$ for band 4 and $2,400 \mathrm{~km} \mathrm{~s}^{-1}$ for band 6 . Observations were taken in an extended configuration with an approximate angular resolution of $\sim 0$ " 4 and $\sim 0$ ". 3 for bands 4 and 6 , respectively. In Table 1 we summarize the observational setup for each band.

\section{Data Reduction and Analysis}

\subsection{OSIRIS: Data Reduction}

The data were reduced with the OSIRIS data reduction pipeline version 3.2, which performs standard near-infrared IFS reduction procedures: it performs dark subtraction, adjusts channel levels, removes crosstalk, identifies glitches, cleans for cosmic rays, extracts spectra, assembles a data cube, and corrects for dispersion. We used the scaled sky-subtraction routine for the Jn1 data cubes, which uses families of atmospheric $\mathrm{OH}$-emission lines between multiple frames for a cleaner subtraction. For Hn3 data we used our own custom skysubtraction routine that scales only the nearest three $\mathrm{OH}$ lines in proximity to the quasar $\mathrm{H} \alpha$ emission line. This produced better residuals than the pipeline's scaled sky routine in $\mathrm{Hn} 3$. Inspection of individual spaxels in the scaled sky-subtracted data cubes still revealed strong $\mathrm{OH}$-sky line residuals, typically over 2-3 spectral pixels in the wings of the $\mathrm{H} \alpha$ emission line. These spectral pixels were linearly interpolated using the slope from the neighboring two pixels around the strong residuals. The telluric spectrum of the calibration star was extracted over the seeing halo, the hydrogen absorption lines removed and its continuum was divided by a $9400 \mathrm{~K}$ blackbody function. The 1D telluric spectrum was normalized and divided into the quasar data cubes. Individual data cubes were then shifted to a common position and combined using a $3 \sigma$ clipping algorithm, which is part of the OSIRIS data reduction pipeline. Finally we applied flux calibration to all quasar data cubes using the telluric corrected standard star spectrum and scaling the $\mathrm{DN} / \mathrm{s}$ /channel to match the expected Jn1 and Hn3 band flux $\left(\right.$ erg s$^{-1} \mathrm{~cm}^{-2}$ ).

\subsection{OSIRIS: PSF Construction and Subtraction}

Both the quasar broad-line emission $(\mathrm{H} \beta$ and $\mathrm{H} \alpha)$ and quasar continuum originate from gas on parsec scales from the SMBH, and are therefore spatially unresolved in our OSIRIS observations. Wavelength channels of the broad-line emission and/or continuum can be used to construct a pristine quasar image that 
Table 1

ALMA Cycle 2 and 3 Observations Summary

\begin{tabular}{|c|c|c|c|c|c|c|c|c|}
\hline Date & Band & $\begin{array}{c}\text { Central Frequencies } \\
(\mathrm{GHz})\end{array}$ & $\begin{array}{c}\text { Integration } \\
\text { Time (minutes) }\end{array}$ & $\begin{array}{l}\text { PWV } \\
(\mathrm{mm})\end{array}$ & $\begin{array}{c}\text { Antennae } \\
\#\end{array}$ & $\begin{array}{l}\text { Beam } \\
\text { Size }\end{array}$ & $\begin{array}{c}\text { Line }^{\mathrm{a}} \\
\sigma / \text { beam (mJy) }\end{array}$ & $\begin{array}{c}\text { Continuum } \\
\sigma / \text { beam }(\mu \mathrm{Jy})\end{array}$ \\
\hline 2015 Aug 6 & 4 & 141.86 & 27.84 & 4.5 & 39 & $0.44 \times 0 . " 41$ & 0.5 & 48 \\
\hline 2016 Sep 9 & 4 & $141.85,140.00,129.97,128.01$ & 24.19 & 2.5 & 37 & $0 . " 39 \times 0 . .30$ & 0.22 & 46 \\
\hline 2016 Sep 17 & 6 & $236.42,234.5,220.99,219.11$ & 19.65 & 0.7 & 38 & $0 . " 28 \times 0$ ". 18 & 0.37 & 44 \\
\hline
\end{tabular}

Note.

${ }^{\mathrm{a}}$ Computed per $34 \mathrm{~km} \mathrm{~s}^{-1}$ channels.

can then be used for PSF subtraction in the reduced data cube. In Vayner et al. (2016) we discuss in greater detail our PSF subtraction routine and its performance on a set of luminous type- 1 radio-quiet quasars at $z \sim 2$. In brief, we used broad emission line/continuum channels that should not overlap with the host galaxy emission with spectral channels offset by $5000-10,000 \mathrm{~km} \mathrm{~s}^{-1}$ from the quasar redshift. We carefully select spectral channels that do not coincide with $\mathrm{OH}$-emission lines or regions of low transparency in the near-infrared. PSFs are generated by combining individual data channels and are scaled to the peak pixel values. This empirical PSF is then subtracted from the entire data cube while rescaling to the peak pixel value of the quasar emission per wavelength channel.

After data reduction, the 100 mas mode in OSIRIS suffers from flux misassignment between adjacent spaxels given its enlarged pupil size in the instrument. The reductions still conserve the integrated flux of the source, but neighboring pixels can receive a $\sim 10 \%$ misassignment of flux. This effect is seen in bright stars $(\mathrm{H}<16 \mathrm{mag})$ with excellent $\mathrm{AO}$ correction, where flux is misassigned from the bright central spaxel to the row above and below the centroid position of the point source. This effect can be easily identified by evidence of spaxels with an inaccurate spectral shape. Our PSF subtraction routine removes a significant portion of flux from the PSF in these spaxels, but they generally have stronger post-PSF subtraction residuals. We masked the affected spaxles with background flux values calculated by taking a standard deviation in a $1^{\prime \prime} \times 1^{\prime \prime}$ sky region. We fit a $2 \mathrm{D}$ Gaussian to the PSF image and measure a FWHM of 0 ". 127 and 0 "! 113 in Hn3 and Jn1, respectively. After PSF subtraction of the quasar data cubes, we smooth the Hn3 and Jn1 data sets to a common resolution with a beam FWHM of 0 ". 2 to improve the signal-tonoise ratio $(\mathrm{S} / \mathrm{N})$ in the diffuse parts of the host galaxy.

\subsection{OSIRIS: Kinematics}

In this section we investigate the kinematics properties of nebular emission lines in the host galaxy of 3C 298. We inspect all individual spectra that overlap between the two observing modes (Jn1 and Hn3), which amounts to approximately 2,640 spectra. An emission line is identified to be real if the peak intensity is at least three times greater than the noise per wavelength channel, and the emission-line dispersion is larger than the instrumental resolution $(0.206 \mathrm{~nm}$ for $\mathrm{Hn} 3$ and $0.174 \mathrm{~nm}$ for $\mathrm{Jn} 1$ ).

A single Gaussian profile provides a good fit to the $486.1 \mathrm{~nm}$ $\mathrm{H} \beta$ emission line that is redshifted into $\mathrm{Jn} 1$ at an observed wavelength of $1186 \mathrm{~nm}$. The 495.9, $500.7 \mathrm{~nm}$ [O III] lines are redshifted into the Jn1 band at an observed wavelength of 1210 and $1221 \mathrm{~nm}$. The 495.9, $500.7 \mathrm{~nm}$ [O III] lines are fit simultaneously, each with a single Gaussian profile. The position and width of the $495.9 \mathrm{~nm}$ line are held fixed to the redshift and width of the $500.7 \mathrm{~nm}$ line at each spaxel. The line ratio between the [O III] lines are held fixed at 1:2.98 (Storey \& Zeippen 2000). The 495.9, 500.7 [O III] lines in several spaxels in the northwest and southeast regions require two Gaussian profiles for a good fit. The [N II] 654.9, $658.5 \mathrm{~nm}$, $\mathrm{H} \alpha 656.3 \mathrm{~nm}$, and [S II] $671.7673 .1 \mathrm{~nm}$ are redshifted into the Hn3 filter. Spaxels with detected $\mathrm{H} \alpha$ and both [N II] emission lines are fit together with three Gaussian profiles. The position and width of the [N II] lines are fixed to the $\mathrm{H} \alpha$ redshift and width, with a flux ratio between [N II] $654.9,658.5 \mathrm{~nm}$ of 1:2.95. Generally, a single Gaussian profile fit to $\mathrm{H} \alpha$ and each [N II] line provides a satisfactory fit $\left(\chi_{R}^{2} \sim 1-2\right)$, with the exception of several spaxels in the southeast region, where an additional broad $\mathrm{H} \alpha$ component is necessary. The $\mathrm{H} \alpha, \mathrm{H} \beta$, and [O III] broad-line components have similar velocity dispersions and offsets. The lower $\mathrm{S} / \mathrm{N}$ of the [S II] doublet make it challenging to fit a single Gaussian profile to each emission line, therefore at each spaxel where [S II] is detected, we fit a single Gaussian profile to the combined signal. Regions where no [N II] or [S II] lines are detected have only a single Gaussian fit to $\mathrm{H} \alpha$. Limits on the [N II] and [S II] lines are derived by inserting a Gaussian profile with the same width as $\mathrm{H} \alpha$ at the expected location of the emission line based on the $\mathrm{H} \alpha$ redshift, with a peak flux twice greater than the standard deviation of the noise. Similarly to a real detection, we integrate the inserted emission line to obtain a limit.

To construct 2D flux maps, we integrate each emission line from $-3 \sigma$ to $+3 \sigma$, where $\sigma$ is derived from the line fit. The error on the line flux is calculated by taking a standard deviation of every three spectral channels and summing in quadrature over the same spectral region where the nebular emission line is integrated. Velocity maps for each emission line are relative to the redshift of the quasar's broad-line region, which is calculated by fitting a single Gaussian profile to the broad $\mathrm{H} \beta$ line constructed by spatially integrating the cube over the seeing halo before PSF subtraction. The velocity dispersion map has the instrumental PSF subtracted out in quadrature using the width of the $\mathrm{OH}$-emission sky lines. The errors on the velocity offsets and dispersions are based on $1 \sigma$ errors associated with the least-squares fit.

In Figure 1 we show the line-integrated emission of $\mathrm{H} \alpha$, [O III], and [S II] in a three-color image composite alongside the [O III] radial-velocity and dispersion maps of 3C 298. A distinct extended broad velocity emission region is cospatial with radio synchrotron emission emanating from extended jet/ lobes. The radio VLA observations are taken from Mantovani et al. (2013; Project code: AJ206). We downloaded fully reduced clean map of 3C 298 at $8485.100 \mathrm{MHz}{ }^{8}$ The centroid

\footnotetext{
8 http://db.ira.inaf.it/aj206-fm/
} 

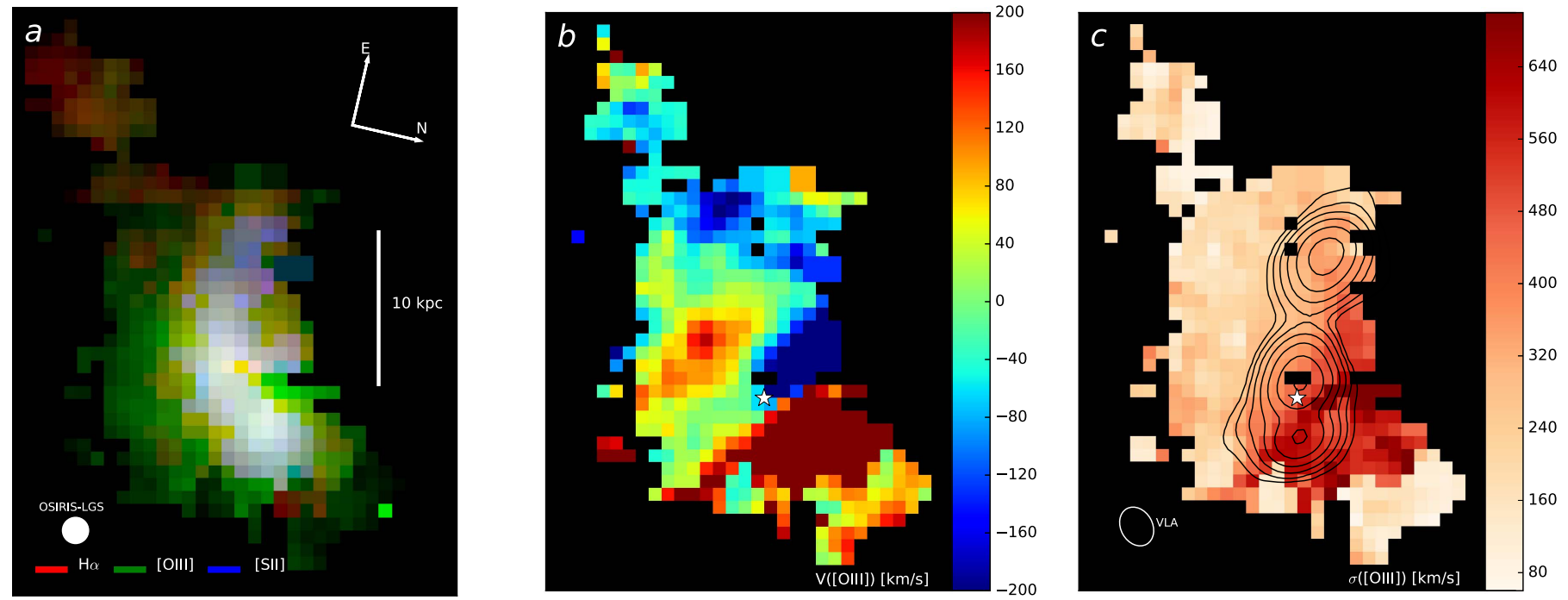

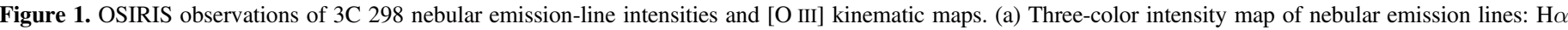

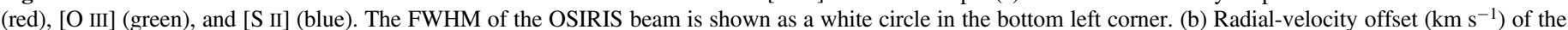

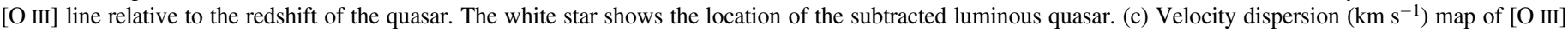

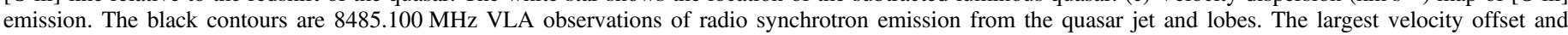

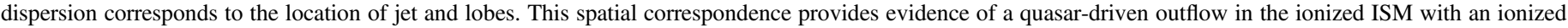
outflow rate of $450-1500 M_{\odot} \mathrm{yr}^{-1}$.

of the point source with the flattest spectral slope is associated with the optical location of the quasar. We extract the image centered on the quasar and rotate to a position angle of $103^{\circ}$ to match our OSIRIS observations.

We generate integrated spectra for three distinct outflow regions identified based on their radial velocity $\left( \pm 400 \mathrm{~km} \mathrm{~s}^{-1}\right)$ and dispersion $\left(V_{\sigma}>500 \mathrm{~km} \mathrm{~s}^{-1}\right)$, see Figure 1. For the purposes of the figure, the northwestern outflow is identified as the redshifted outflow or "Outflow-R," and the northeastern outflow is identified as the blueshifted outflow or "Outflow-B," and the third outflow in the southeastern direction is identified as "AGN-Outflow." The AGN outflow probably belongs to a secondary nucleus in the 3C 298 system becuase of its isolated nature and because the ionized emission does not extend from the quasar and does not coincide with the quasar jet/lobes. The AGN outflow is in close proximity to the dynamical center of a rotating disk in the 3C 298 system that belongs to a second merging galaxy (see Section 3.5 for further discussion). Each spectrum is then fit with multiple Gaussian profiles. Outflow-R is best fit with a combination of two relatively broad $\left(V_{\sigma} \sim 500\right.$ $\mathrm{km} \mathrm{s}^{-1}$ ) Gaussian profiles in the [O III] lines, $\mathrm{H} \alpha$, and [N II], which we interpret as a signature of outflowing gas along the line of sight. The $500.7 \mathrm{~nm}$ [O III] line requires an additional relatively narrow $\left(V_{\sigma} \sim 80 \mathrm{~km} \mathrm{~s}^{-1}\right)$ component for a good fit that has no counterpart in $\mathrm{H} \alpha$, [N II], or [S II]. A faint $\mathrm{H} \beta$ line is detected, and is fit with a single Gaussian component that potentially matches the broad components of [O III] and $\mathrm{H} \alpha$. See the top row of Figure 2 for the integrated spectrum in $\mathrm{J}$ and $\mathrm{H}$ band along with the fit. Components $\mathrm{A}$ (green curve) and $\mathrm{B}$ (red curve) are the broad lines, while component $\mathrm{C}$ (blue curve) is the narrow line found only in [O III]. The white contour in the right column shows the region over which the data cube is spatially integrated. The spectrum of the blueshifted outflow $($ Outflow-B) region is best fit with a single broad Gaussian component in $\mathrm{H} \beta$, [O III], $\mathrm{H} \alpha$, and [N II]; the second row of Figure 2 shows the spectra along with the fit. In the redshifted outflow region the lines are very broad, so the [S II] doublet is blended, making it very hard to fit the individual lines. The lines are slightly narrower in the blueshifted outflow region, and each emission line in [O III] and $\mathrm{H} \alpha$ requires only a single Gaussian component. This made it possible to fit the [S II] doublet using a single Gaussian profile for each emission line.

We construct a high $\mathrm{S} / \mathrm{N}$ spectrum over the entire $A G N$ Outflow region. $\mathrm{H} \alpha$ and [O III] are fit with two components: a broad-line blueshifted Gaussian for the outflow, and a narrow component that we interpret as part of the AGN/quasar narrow-line region. $\mathrm{H} \beta,[\mathrm{N} I I]$, and [S II] are fit with a single Guassian narrow-line component.

\subsection{OSIRIS: Nebular Emission-line Diagnostics}

In this section we investigate potential ionizing sources for distinct regions of the host galaxy. Line ratio maps are constructed by taking ratios of integrated flux maps [O III], $\mathrm{H} \alpha$, $[\mathrm{N} \mathrm{II}]$, and [S II]. The $\mathrm{H} \beta$ line is only detected in a small number of individual spaxels; sensitivity and dust obscuration prohibits us from detecting this line over similar sized regions to other detected nebular emission lines. We construct an $\mathrm{H} \beta$ map over the region where $\mathrm{H} \alpha$ is detected by assuming case-B recombination $\left(F_{\mathrm{H} \beta}=F_{\mathrm{H} \alpha} / 2.89\right)$.

We create three line ratio maps $\log ([\mathrm{O} \mathrm{III}] / \mathrm{H} \beta), \log ([\mathrm{N} \mathrm{II}] /$ $\mathrm{H} \alpha)$, and $\log ([\mathrm{S} \mathrm{II}] / \mathrm{H} \alpha)$. In Figure 3 we plot the line ratios on a standard BPT diagram, $\log ([\mathrm{O} \mathrm{III}] / \mathrm{H} \beta)$ versus $\log ([\mathrm{N} \mathrm{III}] / \mathrm{H} \alpha)$. Empirical (Kauffmann et al. 2003) and theoretical (Kewley et al. 2001) curves separating photoionization by O-type stars versus quasar ionization are shown. Generally, values that lie above these curves represent photoionization by an AGN, while points below represent photoionization by newly formed O-type stars, tracing regions of active/recent star formation. Points on the BPT diagram are color coded to match the line ratio map of $\log ([\mathrm{O} \mathrm{III}] / \mathrm{H} \beta$ ) (middle panel in Figure 3$)$. The $1^{\prime \prime}$ central region (red and orange in Figure 3) is mainly photoionized by the quasar in an extended narrow-line region (ENLR), as shown by very high $\log ([\mathrm{O} \mathrm{III}] / \mathrm{H} \beta) \gtrsim 0.8$ values. $658.5[\mathrm{~N}$ II] is detected over the red region in individual 

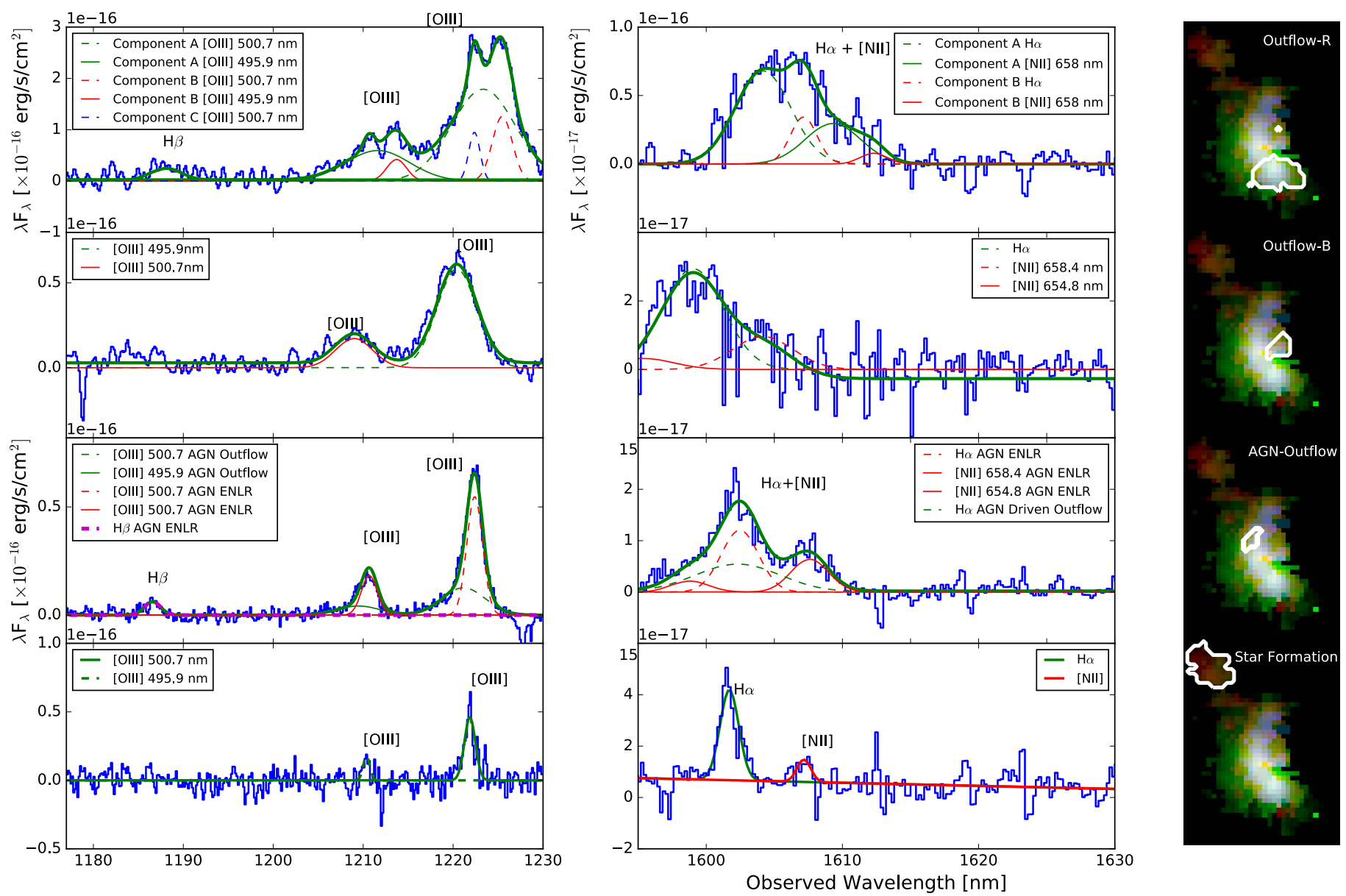

Figure 2. OSIRIS near-infrared spectra for distinct photoionized regions in the host galaxy of 3C 298. J- and H-band spectra are shown in the left and middle panels, respectively, with observed wavelengths $(\mathrm{nm})$ and calibrated flux $\left(\mathrm{F}_{\lambda}\right)$. The three-color composite image (right) shows the regions over which the spectra were extracted. Multiple Gaussian functions are fit to each of the emission lines: $\mathrm{H} \beta$, [O III], $\mathrm{H} \alpha$, and [NII] in all the spectra. The green curves show the combined fit to each spectrum, and each dotted line represents an individual component of the fit. The redshifted outflow region is best fit with a double Gaussian component (labeled A and B in the top panel) to each nebular emission line, which suggests a conical expansion of the gas along the line of sight. The blueshifted outflow region is best modeled with a single Gaussian component to each emission line. The presence of broad, blueshifted emission lines in [O III] and H $\alpha$ (labeled "AGN Outflow," third panel from the top), indicating outflowing gas, is roughly coincident with the dynamical center of the secondary galaxy in the merger. This outflow region is not associated with strong star formation, based on line ratio diagnostics in Figure 3. The star-forming region is best fit with a single narrow Gaussian component to each emission line.

spaxels, while over the orange region, we place a $2 \sigma$ flux limit. Even within the limits, the orange region falls in the AGN photoionzation portion of the BPT diagram due to very high $\log ([\mathrm{O} \mathrm{III}] / \mathrm{H} \beta)$ values. The star formation region (blue) has $\operatorname{low} \log ([\mathrm{O} \mathrm{III}] / \mathrm{H} \beta)<0.8$ and $\log ([\mathrm{N} \mathrm{II}] / \mathrm{H} \alpha)<0.5$ values, corresponding to ionization from newly formed stars. We generate a spectrum over the Star Formation region and easily identify and fit a single Gaussian to the [O III], $\mathrm{H} \alpha$, [N II], and a $\mathrm{H} \beta$ line with an $\mathrm{S} / \mathrm{N}$ of 2 identified (bottom panel of Figure 2).

Star-forming regions are spatially offset from the large outflows, and their kinematics and morphologies agree with a potential tidal feature induced by the merging galaxies. Outflow regions (yellow and green) correspond to the highest $\log ([\mathrm{O} \mathrm{III}] / \mathrm{H} \beta)$ line ratios. These regions are inconsistent with ionization by young stars, and are photoionized by quasars and shocks. Gas in outflow regions is moving at sufficiently high velocity, so the Mach number of the wind is high, and strong radiative shocks are expected. Using shock models from Allen et al. (2008), we find that a large portion of observed line ratios agree with shock models for gas with an electron density between $10^{2}-10^{3} \mathrm{~cm}^{-3}$ and shock velocities of $1000 \mathrm{~km} \mathrm{~s}^{-1}$. In Figure 4 we plot the $\log ([\mathrm{O} \mathrm{III]} / \mathrm{H} \beta)$ versus $\log ([\mathrm{S} \mathrm{II}] / \mathrm{H} \alpha)$ values over regions where [S II] was detected in individual spaxels. We overlay shock models on this BPT diagram. A large fraction of the nebular line ratios in the redshifted outflow region (green) and blueshifted outflow region (yellow) are consistent with these models. Dark red/blue lines in the model represent higher velocity gas and stronger magnetic parameter (see Allen et al. 2008 for further details). Furthermore, the region that is photoionized by AGN shows a spatial profile that drops off as $1 / R^{2}$ in nebular emission, while in the region where we see powerful outflows, the profile is more complex, suggesting multiple ionizing sources. Both regions show similar extinction values, as we discuss below.

In order to investigate how dust can alter our photoioniziation measurements, we construct a high $\mathrm{S} / \mathrm{N}$ spectrum over the ENLR (red in Figure 3) to estimate the amount of dust obscuration from the $\mathrm{H} \alpha / \mathrm{H} \beta$ ratio. Using the Calzetti et al. (2000) law, we find an average $A_{V}=0.07$ over the ENLR (the red region in Figure 3), based on an $\mathrm{H} \alpha / \mathrm{H} \beta$ ratio of 2.9. This would on average increase the ratio of $\log ([\mathrm{O}$ III $] / \mathrm{H} \beta$ ) only by a factor of 1.05 for the ENLR; this is not large enough to alter our results. We find that in portions of the ENLR and redshifted outflow region, the $A_{v}$ value is consistent with zero extinction 

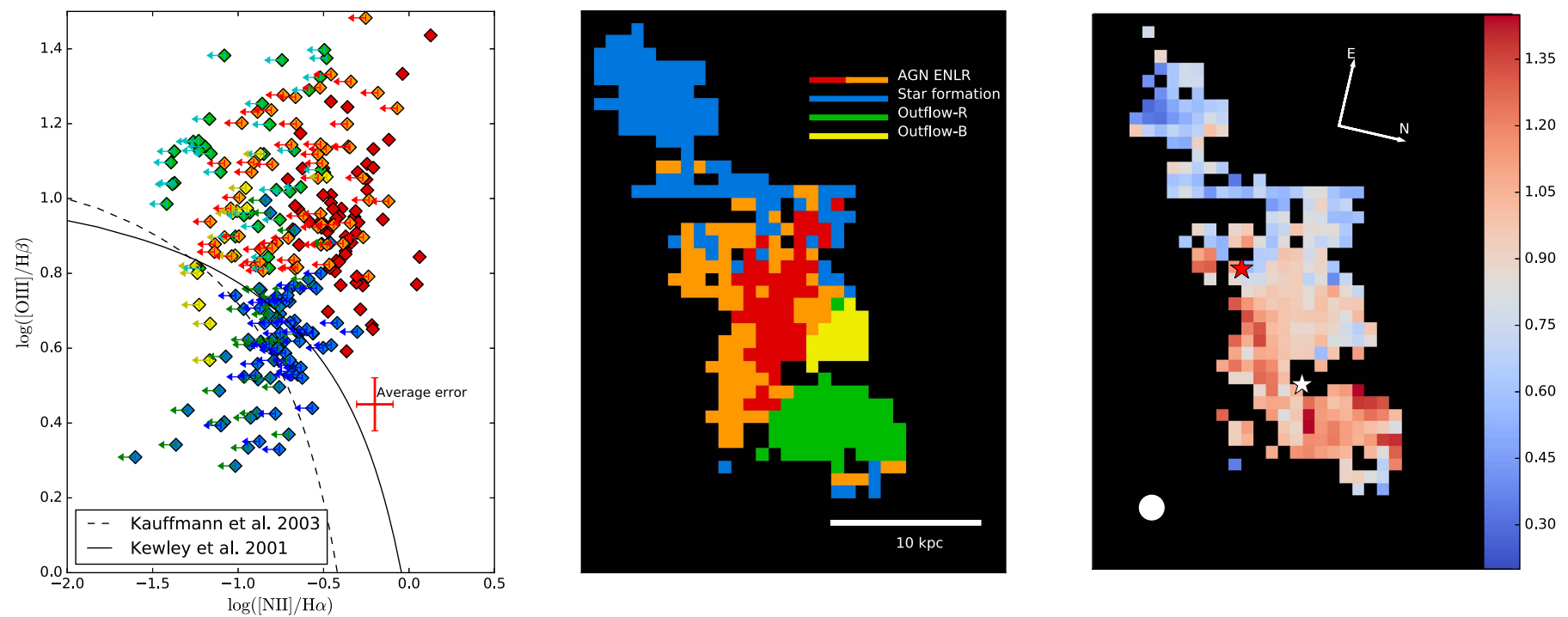

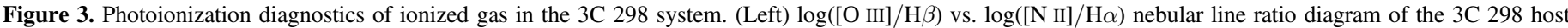

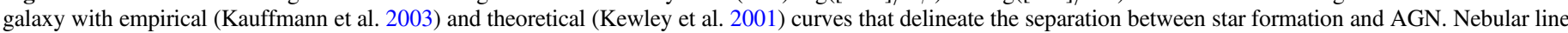

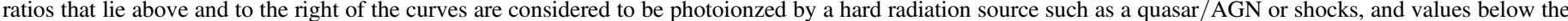

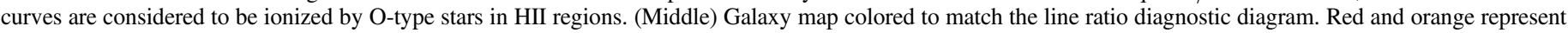

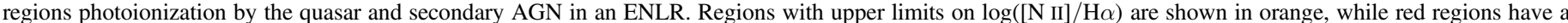

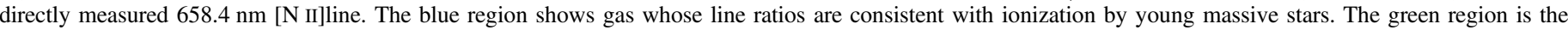

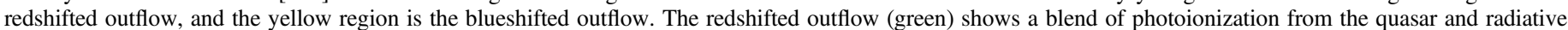

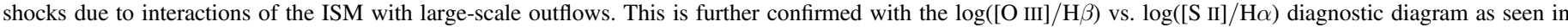

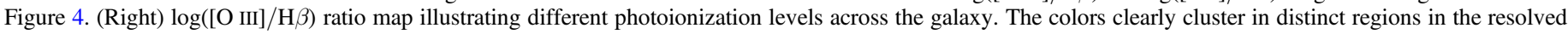

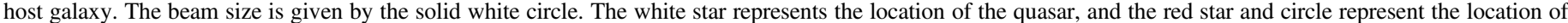
the dynamical center of the secondary galaxy, where a potential secondary obscured AGN exists.
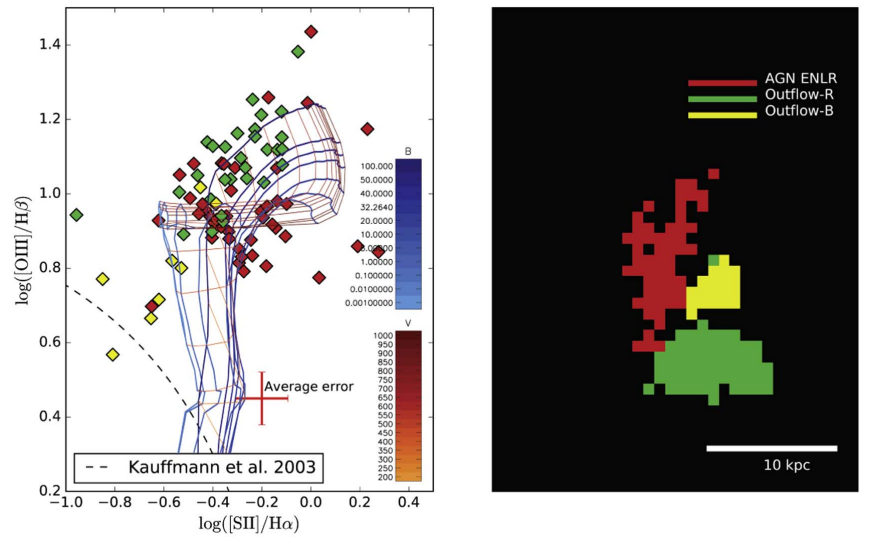

Figure 4. Photoionzation diagnostics of ionized gas in the 3C 298 system (Left) Diagram of $\log ([\mathrm{O} \mathrm{III}] / \mathrm{H} \beta)$ vs. $\log ([\mathrm{S} \mathrm{II}] / \mathrm{H} \alpha)$ with an empirical dashed curve (Kauffmann et al. 2003) that separates photoionization of star-forming regions and AGN. The theoretical position of the shocked ionization is overlaid on the BPT diagram (Allen et al. 2008). Dark red/blue lines in the model represent higher velocity gas and stronger magnetic parameter. (Right) Colored values match the spatial regions of the host galaxy, where the green and yellow points show the redshifted and blueshifted outflow regions. Ionization in these outflow regions is due to shocks moving at speeds of $\sim 1000 \mathrm{~km} \mathrm{~s}^{-1}$. The red region is consistent with values primarily ionized by AGN.

in the ionized gas. Similar results are found over the outflow regions, suggesting negligible extinction in the ionized gas. Over the star-forming region (blue region in Figure 3), we measure an $A_{V}$ value of 1.2 based on an $\mathrm{H} \alpha / \mathrm{H} \beta$ ratio of 4.0. The observed non-detection of $\mathrm{H} \beta$ in individual spaxels is consistent with the derived integrated extinction value and the expected $\mathrm{H} \beta \mathrm{S} / \mathrm{N}$ relative to [O III] and $\mathrm{H} \alpha$ emission lines.

\subsection{OSIRIS: Velocity Field Modeling}

To the southeast of the quasar, there is a kinematic feature resembling a rotating galactic disk that is offset by $\sim 200 \mathrm{~km} \mathrm{~s}^{-1}$ from the quasar BLR, with a projected rotational velocity of $\pm 170 \mathrm{~km} \mathrm{~s}^{-1}$. In this section we outline the modeling done on the radial-velocity map of [O III] emission to confirm the rotating-disk nature of this feature. We isolate this feature for the disk fitting, using a box size of 0 !! $7 \times 1$ !! 7 centered on the gradient feature in the [O III] radial-velocity map. The boxed region is selected to exclude any strong broad emission from the conical outflow. The [O III] velocity field is modeled by fitting a 2D arctangent disk model given by

$$
V(r)=\frac{2}{\pi} V_{\max } \arctan \left(\frac{r}{r_{\mathrm{dyn}}}\right),
$$

where $V(r)$ is rotation velocity at radius $r$ from the dynamical center, $V_{\max }$ is plateau velocity, and $r_{\text {dyn }}$ is the radius at which the arctangent function has a turn over to a decreasing slope. The measured line-of-sight velocity from our observations relates to $V(r)$ as

$$
V=V_{0}+\sin i \cos \theta V(r),
$$

where

$$
\cos \theta=\frac{\left(\sin \phi\left(x_{0}-x\right)\right)+\left(\cos \phi\left(y_{0}-y\right)\right)}{r} .
$$

The radial distance from the dynamical center to each spaxel is given by

$$
r=\sqrt{\left(x-x_{0}\right)^{2}+\left(\frac{y-y_{0}}{\cos i}\right)^{2}},
$$



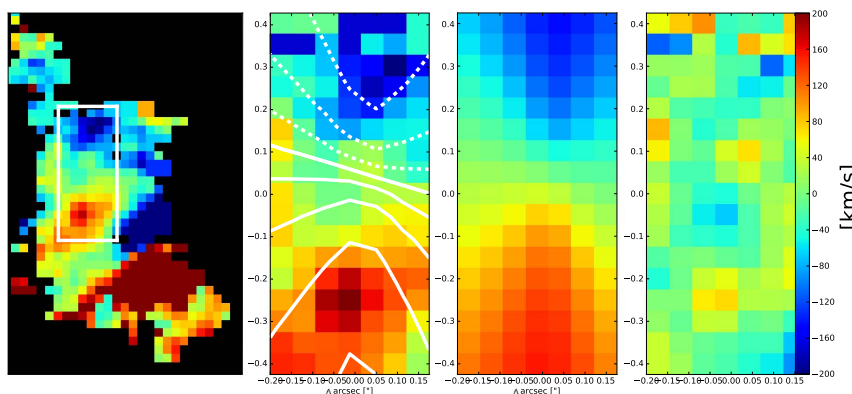

Figure 5. Observed and modeled galactic rotation disk, detected in the nebular [O III] line in the 3C 298 system. (Left) Entire [O III] radial-velocity map with the overlaid region we used for the modeled disk fit. (Middle left) Observed [O III] velocities with the spider diagram overlaid from the disk model. (Middle right) Best-fit 2D disk model. (Right) Residual map of the observed velocities subtracted by the disk model fit. The dynamical center is at 0,0 .

where $x_{0}, y_{0}$ is spaxel location of the dynamical center, $V_{0}$ is velocity offset at the dynamical center relative to the redshift of the quasar broad-line region, $\phi$ is the position angle in spaxel space, and $i$ is the inclination of the disk.

We fit the seven-parameter velocity field model to a selected region of our observed [O III] velocity map using a nonlinear leastsquares routine. The selected box region tries to exclude broad emission from the extended quasar outflow and a tidal feature that seems to show its own distinct kinematic structure. Our best fit has a $\chi_{R}^{2}=0.7$ with the following values: $x_{0}, y_{0}=-0$ ". $49,+0$ ". 94 relative to the centroid of the quasar, $V_{\max }=209 \pm 33 \mathrm{~km} \mathrm{~s}^{-1}$, $r_{\text {dyn }}=2.2 \pm 0.98 \mathrm{kpc}, \quad i=72^{\circ} \pm 4^{\circ}, \phi=-109^{\circ} \pm 6^{\circ}$, and $V_{0}=170 \pm 9 \mathrm{~km} \mathrm{~s}^{-1}$ relative to the redshift of the quasar broadline region. Figure 5 shows the region selected for disk fitting with the modeled 2D velocity profile and residuals between the model and observed velocities. The disk is offset from the quasar by 1 ". 06 or $\sim 9 \mathrm{kpc}$, which is evidence of a merging disk system in 3C 298. We obtain a dynamical mass of $1.3 \pm 0.8 \times 10^{10} M_{\odot}$ within a radius of $r_{\mathrm{dyn}}=2.2 \mathrm{kpc}$. The dynamical center of this disk is in the vicinity of the isolated AGN outflow identified in Figure 2. With currently available archival Chandra ACIS observations, we are unable to explore the X-ray properties of this secondary AGN candidate. Unfortunately, there is strong asymmetry in the Chandra PSF along this position angle from the quasar. Future Chandra observations at a specific spacecraft roll angle will allow us to search for X-ray emission from this secondary AGN and explore the potential dual AGN nature of this system.

\subsection{ALMA: Data Reduction}

Data reduction was performed using CASA (Common Astronomy Software Applications; McMullin et al. 2007) versions 4.4 and 4.7. At the observed frequencies, synchrotron emission from the quasar dominates molecular $\mathrm{CO}$ or dust emission, and has sufficient $\mathrm{S} / \mathrm{N}$ to perform self-calibrations directly on the science source. We used the CASA CLEAN function to establish a model for the synchrotron continuum through several interactive runs with clean masks centered on high $\mathrm{S} / \mathrm{N}$ features. Cleaning is performed with Briggs weighting using a robust value of 0.5 with a pixel scale of 0 ."05. We used the gaincal function to perform phase corrections. The self-calibrated data were then cleaned again with further phase corrections until we no longer saw a significant improvement in the $\mathrm{S} / \mathrm{N}$ on the continuum. The final root mean square (rms) improved by a factor of 6-8 in the continuum images. We perform continuum subtraction in UV space by fitting a first-order polynomial to channels free of line emission from the host galaxy, and subtract this fit from the rest of the channels.

Data cubes were imaged using clean with a plate scale of 0 ." 05 per pixel, a spectral channel size of $34 \mathrm{~km} \mathrm{~s}^{-1}$, and natural weighting to improve the $\mathrm{S} / \mathrm{N}$ in the diffuse structure of the host galaxy at the cost of angular resolution. For cycle 3 data, clean masks were placed on $\mathrm{CO}$ emission with $\mathrm{S} / \mathrm{N}>5$ with the same mask applied to all channels. The resulting beam sizes and rms values are reported in Table 1.

We find that the cycle 2 band 4 data cube is a factor of 2 noisier due to poorer weather conditions at the time of observations. Faint emission is detected with a peak $\mathrm{S} / \mathrm{N}$ of $3.6 \sigma$ for $\mathrm{CO}(J=3-2)$ emission over a number of spaxels in the east/northeast direction from the quasar. Applying UV tapering to a resolution of 0 ". $98 \times 0$ ". 8 with Briggs weighting (robust $=0.5$ ) improves the $\mathrm{S} / \mathrm{N}$ to 5 , but the line is not spatially resolved. For the analysis of $\mathrm{CO}(J=3-2)$, we therefore defer to cycle 3 data taken under superior weather conditions.

We align the ALMA and OSIRIS data cubes by matching the position of the optical quasar emission to the unresolved quasar synchrotron emission centroid in the continuum map. The centroid of the quasar matches the location of the unresolved point source with the flattest spectra seen using high-resolution centimeter observations of 3C 298 with MERLIN (component B1/B2 in Fanti et al. 2002). This location is typically associated with the quasar core. Finally, we rotate the ALMA data cube to a position angle of $103^{\circ}$ to match the OSIRIS observations.

\subsection{ALMA: Analysis}

In this section we describe the analysis of resolved $\mathrm{CO}(3-2)$ and (5-4) 3D spectroscopy. Initial inspection revealed a high $\mathrm{S} / \mathrm{N} \gtrsim 5$ detection of $\mathrm{CO}(J=3-2)$ and $(J=5-4)$ emission. We extracted spectra over the beam size centered on the detected regions. We then collapsed the data cube over spectral channels where the $\mathrm{CO}$ features are detected, and constructed $\mathrm{S} / \mathrm{N}$ maps by dividing the integrated emission by the standard deviation computed in an empty-sky region. Spaxels that showed an emission line with an $\mathrm{S} / \mathrm{N} \geqslant 3$ were fit with a Gaussian profile. We constructed a flux map by integrating over the line $(-3 \sigma$ to $+3 \sigma)$. We created a velocity map by computing the Doppler shift of the emission-line centroid relative to the redshift $(z=1.439)$ of the broad-line region (calculated from $\mathrm{H} \beta$ ), and generated a velocity dispersion map from the Gaussian fit. Figure 6 shows the CO emission, radial velocity, and dispersion maps.

\subsubsection{Molecular Gas Velocity Field Modeling}

In this section we outline the modeling performed on the $\mathrm{CO}(3-2)$ radial-velocity map to derive a dynamical mass for the inner $\sim 2 \mathrm{kpc}$ of the host galaxy. We find that the majority of the $\mathrm{CO}$ emission is concentrated at the spatial location of the quasar and has a secondary peak offset by $16 \mathrm{kpc}$ that is spatially coincident with the recent star formation from the OSIRIS BPT analysis (see Figure 3 and Section 3.4). CO (3-2) emission concentrated on the quasar shows a distinct velocity gradient resembling a rotating disk, with an extent of about 0.18 with a velocity difference of $\pm 150 \mathrm{~km} \mathrm{~s}^{-1}$. Similar to Section 3.5 , we model the molecular disk with a hyperbolic arctangent function 

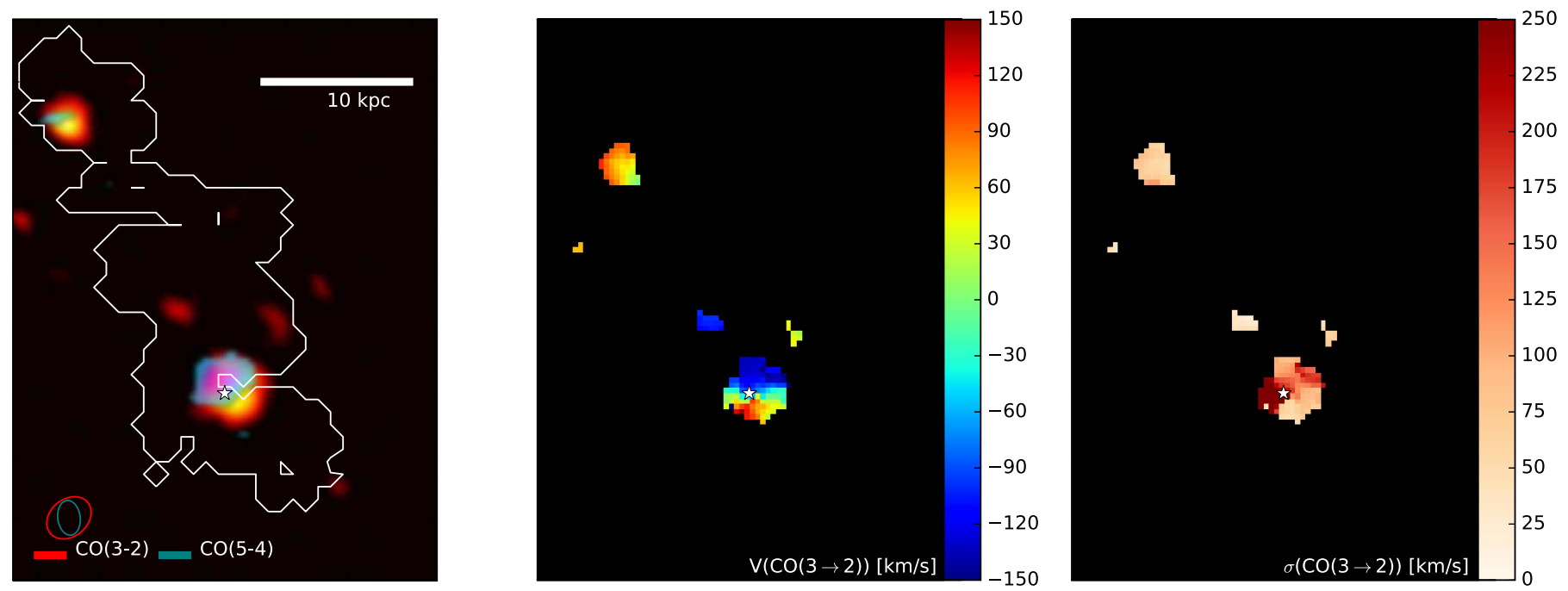

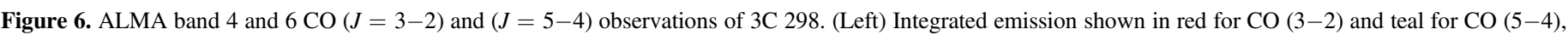

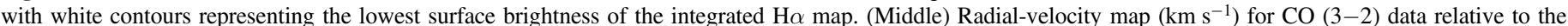

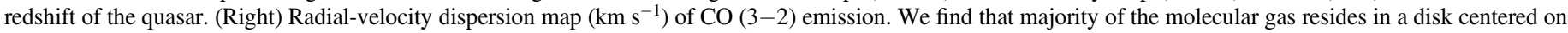

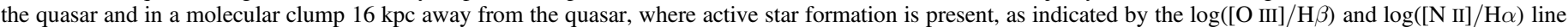
ratios (blue region in Figure 3). The synthesized beam is shown in the lower left corner. The white star marks the location of the quasar.

and derive the following properties about the disk: $\chi_{R}^{2}=$ 0.6: $x_{0}, y_{0}=0 . \prime 05,0$ " 0 relative to the centroid of the quasar, $V_{\max }=392 \pm 65 \mathrm{~km} \mathrm{~s}^{-1}, \quad r_{\text {dyn }}=2.1 \pm 0.9 \mathrm{kpc}, \quad i=54^{\circ} .37 \pm$ $6.4, \phi=5^{\circ} .3 \pm 1.28$, and $V_{0}=-13.0 \pm 3.15 \mathrm{~km} \mathrm{~s}^{-1}$. Fits of the disk along with the residuals are shown in Figure 7. We derive a dynamical mass $1.7 \pm 0.9 \times 10^{10} M_{\odot}$ within $r_{\text {dyn }}=2.1 \mathrm{kpc}$ using the disk inclination derived from the velocity modeling.

Alternatively, we collapsed the data cube along the spectral direction from $-150 \mathrm{~km} \mathrm{~s}^{-1}$ to $+150 \mathrm{~km} \mathrm{~s}^{-1}$ to produce a CO (3-2) image of the molecular disk. Using a 2D Gaussian, we obtained a major axis value of 0 ". $374 \pm 0$ " 05 and a minor axis of 0 ." $155 \pm 0$." 098 corrected for the beam size. Assuming the molecular disk can be approximated by an oblate spheroid, we derived the inclination angle using the following formula (Holmberg 1946):

$$
i=\cos ^{-1} \sqrt{\frac{(b / a)^{2}-q_{0}^{2}}{1-q_{0}^{2}}},
$$

where $\mathrm{a}$ and $\mathrm{b}$ are the major and minor axes, and $q_{0}$ is the axial ratio for an edge-on disk taken to be 0.13 . We derived a dynamical mass of $1.0 \pm 0.5 \times 10^{10} M_{\odot}$ using a major axis radius of $1.6 \pm 0.215 \mathrm{kpc}$ and an inclination angle of $66^{\circ} .6 \pm 17.8$. The dynamical masses and disk inclination angles obtained with these two methods agree within the errors. With two distinct galactic disks (see Section 3.5) in the 3C 298 system, we find evidence for an intermediate- to late-stage merger.

Shen et al. (2011) determined a single-epoch black hole mass for 3C 298 using three calibration methods: $10^{9.57 \pm 0.03} M_{\odot}$ using the MgII-black hole mass relationship calibrated from their study, $10^{9.37 \pm 0.03} M_{\odot}$ using the Vestergaard \& Osmer (2009) calibration, and $10^{9.56 \pm 0.01} M_{\odot}$ using the McLure \& Dunlop (2004) relation. The systematic differences between black hole mass measurements are typically due to differing quasar samples and the properties of the emission lines (e.g., $\mathrm{H} \beta$ ) that were used to calculate the SMBH mass.
With both the derived dynamical and black hole mass, we can compare the nuclear region of 3C 298 to the local black hole $M_{\text {bulge }}-M_{\mathrm{BH}}$ relationship. In Figure 8 we plot the 3C 298 black hole and bulge dynamical mass relative to the local scaling relation from Häring \& Rix (2004), Sani et al. (2011), and McConnell \& Ma (2013). Compared to all three relationships, the 3C 298 bulge dynamical mass is either $\sim 2-2.5$ orders of magnitude lower than what is expected, or the black hole is $\sim 2$ orders of magnitude higher than what is expected for its bulge mass. If we assume that the 3C 298 bulge is more extended than what is measured by ALMA, then we can use the tidal feature at $21.5 \mathrm{kpc}$ as an estimate for the total enclosed mass. To do this, we integrate all spaxels with a detected CO (3-2) line and remove the outflow contribution by simultaneously fitting a broad and a narrow Gaussian component to the outflow region. We measure a maximum line width of $369.3 \mathrm{~km} \mathrm{~s}^{-1}$ at $90 \%$ intensity, and find a maximum circular velocity of $184.6 / \sin (i) \mathrm{km} \mathrm{s}^{-1}$ at $21.5 \mathrm{kpc}$ from the quasar. Using this maximum circular velocity, we obtain a total enclosed mass at this radius of $\sim 2.7 \times 10^{11} M_{\odot}$, assuming the smallest measured inclination angle from the molecular disk. This value should be considered as a maximum limit for the total enclosed mass of the 3C 298 system. This enclosed dynamical mass at $21.5 \mathrm{kpc}$ from the quasar still places 3C 298 an order of magnitude above the local scaling relation. The radius used for this calculation is also significantly larger than the typical bulge radius at this redshift (Bruce et al. 2014; Sachdeva et al. 2017), and there is no guarantee that the entire enclosed stellar mass will end up in the newly created bulge when the merger is completed. This implies a delay in growth of the galactic bulge for the $3 \mathrm{C}$ 298 host galaxy with respect to its SMBH. Averaging SMBH masses and dynamical masses determined for 3C 298, we obtain the following values: $M_{\text {dyn,bulge }}=1.35 \pm 0.5 \times 10^{10}$, and $M_{\mathrm{SMBH}}=3.23 \pm 1.1 \times 10^{9}$. Errors are added in quadrature with the SMBH mass standard deviation included to address the systematic uncertainty associated with using various calibration methods. 

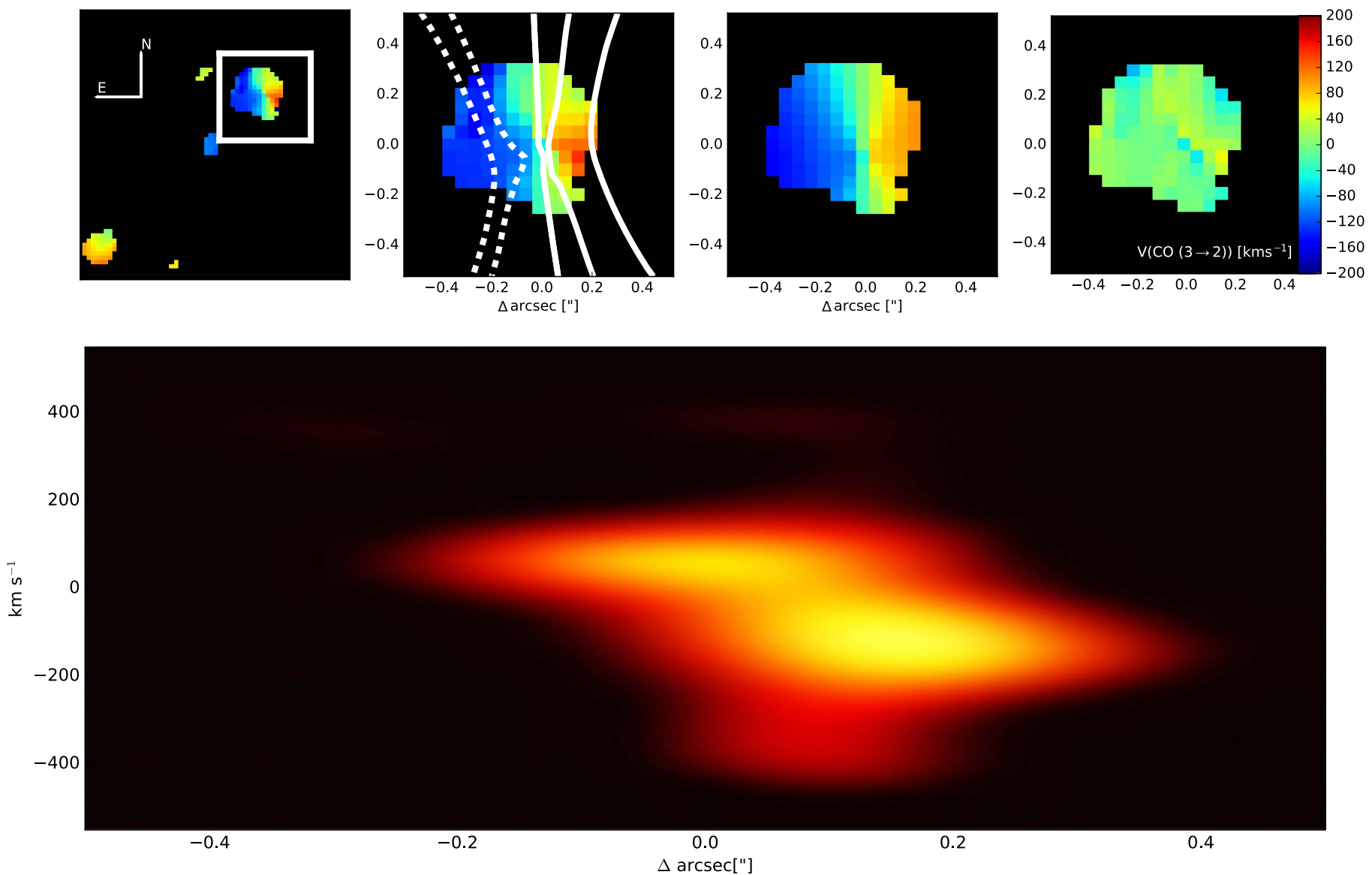

Figure 7. (Top left) Velocity field modeling of the molecular disk. $\mathrm{CO}(3-2)$ radial-velocity map with a box highlighting the location of the molecular disk. (Middle left) Boxed region with a spider diagram of the disk model. (Middle right) Best-fit rotating-disk model. (Right) Residuals between the observed velocity and model fit. The quasar is located at 0,0 . The fitting was made on data at the ALMA observation position angle of $0^{\circ}$ to avoid any pixel interpolation that may cause a difference in the fitting results. (Bottom) Position-velocity (PV) diagram along the major axis of the ALMA disk, which illustrates the the velocity field and plateau velocity on a kiloparsec scale. Broad blueshifted emission that is not associated with the disk rotation is observed in the PV diagram from -400 to $200 \mathrm{~km} \mathrm{~s}^{-1}$ as the large molecular outflow.

\subsubsection{Molecular Gas Mass}

In this section we derive the molecular gas mass from the spatially integrated $\mathrm{CO}(3-2)$ and $(5-4)$ emission presented in Table 2.

In order to measure the molecular gas in the 3C 298 system, we convert the $\mathrm{CO}(3-2)$ flux into $L_{\mathrm{CO}(3-2)}^{\prime}$ using the following equation from Carilli \& Walter (2013),

$$
\begin{gathered}
L_{\mathrm{CO}(3-2)}^{\prime}=3.25 \times 10^{7} \mathrm{~S}_{\mathrm{CO}(3-2)} \Delta \mathrm{v} \\
\times \frac{D_{\mathrm{L}}^{2}}{(1+z)^{3} \nu_{\mathrm{obs}}^{2}} \mathrm{~K} \mathrm{~km} \mathrm{~s}^{-1} \mathrm{pc}^{2} .
\end{gathered}
$$

We convert into $\mathrm{CO}(1-0)$ luminosity $\left(L_{\mathrm{CO}(1-0)}^{\prime}\right)$ using an $L_{\mathrm{CO}(3-2)}^{\prime} / L_{\mathrm{CO}(1-0)}^{\prime}$ ratio of 0.97 (Carilli \& Walter 2013). Using the typical $\alpha_{\mathrm{CO}}$ value from nuclear star bursts and quasars $\left.\left(0.8 \mathrm{~K} \mathrm{~km} \mathrm{~s}^{-1} \mathrm{pc}^{2}\right)^{-1}\right)$, we derive a total molecular gas mass equal to $8.75 \pm 0.4 \times 10^{9} M_{\odot}$. The total molecular gas includes all regions with a fitted CO (3-2) line in Figure 6. The molecular gas disk contains $6.6 \pm 0.36 \times 10^{9} M_{\odot}$, while the active star formation region that lies $16 \mathrm{kpc}$ away contains $1.44 \pm 0.14 \times 10^{9} M_{\odot}$. We measure a $2 \sigma$ molecular gas limit of $1 \times 10^{9} M_{\odot}\left(\frac{\alpha_{\mathrm{CO}}}{0.8}\right)\left(\frac{V_{\mathrm{FWHM}}}{200 \mathrm{~km} \mathrm{~s}^{-1}}\right)$ per beam. This corresponds to a molecular gas surface density of
$104 M_{\odot} \mathrm{pc}^{-2}\left(\frac{\alpha_{\mathrm{CO}}}{0.8}\right)\left(\frac{V_{\mathrm{FWHM}}}{200 \mathrm{~km} \mathrm{~s}^{-1}}\right)$. These estimates are valid for the inner few arcseconds around the phase center (quasar).

The CO (3-2) and (5-4) emission in some spaxels shows relatively broad $\left(V_{\sigma}>270 \mathrm{~km} \mathrm{~s}^{-1}\right)$ emission, greater than the escape velocity $\sqrt{2} V_{\text {rotational }} \sim 270 \mathrm{~km} \mathrm{~s}^{-1}$ at the observed edge of the rotating disk. In the position-velocity diagram (Figure 7), broad blueshifted emission is seen, with velocities ranging from -400 to $200 \mathrm{~km} \mathrm{~s}^{-1}$. This emission spatially lies away from the ordered $\pm 150 \mathrm{~km} \mathrm{~s}^{-1}$ rotation profile. When we integrate over these spaxels, the $\mathrm{CO}(3-2)$ and (5-4) emission profiles resemble both an outflow with broad emission and narrow emission that is likely emanating from the molecular disk. We fit a double Gaussian component to the $\mathrm{CO}(3-2)$ line and a single Gaussian to the CO (5-4) data. We measure a FWHM of $624 \pm 49 \mathrm{~km} \mathrm{~s}^{-1}$ for the broad-line emission in CO (3-2) and $687 \pm 18 \mathrm{~km} \mathrm{~s}^{-1}$ in $\mathrm{CO}(5-4)$. The broad emission line represents either an extended outflow originating from the nuclear region of the 3C 298 quasar or from the molecular disk itself. Figure 9 shows spectra of the $\mathrm{CO}(3-2)$ and (5-4) emission regions with their corresponding Gaussian fits. The broad emission in both lines is predominantly found on the blueshifted side of the rotating disk. The total molecular gas in the outflow is $3.3 \pm 0.1 \times 10^{9} M_{\odot}$. In Section 4 we measure the molecular gas outflow rate, kinetic energy, and momentum to understand its impact on the galaxy. 


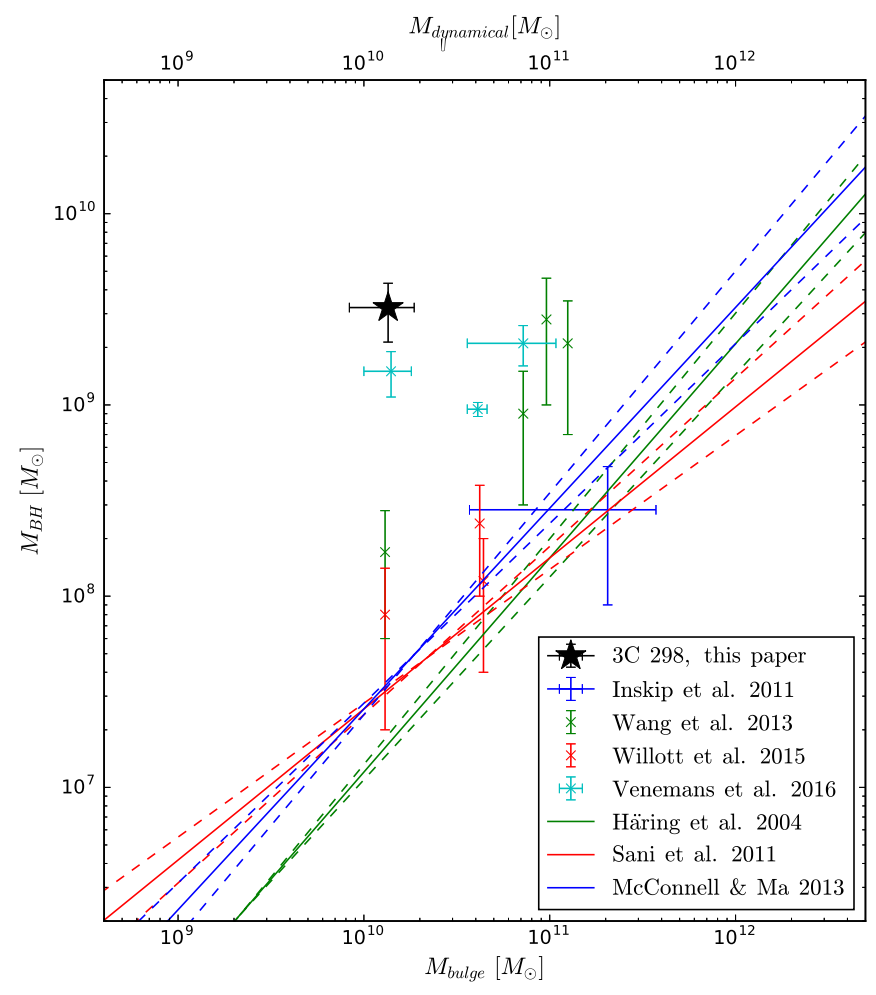

Figure 8. Local SMBH vs. galactic bulge dynamical mass relationship compared to measured values for 3C 298. The black star represents the average dynamical mass derived from the intensity and kinematic molecular gas disk modeling and the average black hole mass derived with the relationships reported by McLure \& Dunlop (2004), Vestergaard \& Osmer (2009), Shen et al. (2011). Errors are added in quadrature from the various methods used to derive the dynamical mass of the disk and the black hole mass. Local scaling laws from various studies (Häring \& Rix 2004; Sani et al. 2011; McConnell \& Ma 2013) are shown as solid lines, and their respective $1 \sigma$ errors are shown with dotted curves. The observed black hole mass and dynamical mass of the bulge of 3C 298 lies $~ 2-2.5$ orders of magnitude away from the local scaling relation. Points from the literature (Wang et al. 2013; Willott et al. 2015; Venemans et al. 2016) for $z>5.7$ quasars are also included. There is a line of evidence that some of these high-redshifts systems also lie off the local scaling laws.

Table 2

Measured Molecular-line Intensities

\begin{tabular}{lcr}
\hline \hline Region & $I_{\mathrm{CO}(3-2)}$ & \multicolumn{1}{c}{$I_{\mathrm{CO}(5-4)}$} \\
\hline Molecular Disk $^{\mathrm{a}}$ & $0.63 \pm 0.035 \mathrm{Jy} \mathrm{km} \mathrm{s}^{-1}$ & $1.26 \pm 0.063 \mathrm{Jy} \mathrm{km} \mathrm{s}^{-1}$ \\
Tidal Feature $^{\mathrm{a}}$ & $0.13 \pm 0.013 \mathrm{Jy} \mathrm{km} \mathrm{s}^{-1}$ & $0.1 \pm 0.025 \mathrm{Jy} \mathrm{km} \mathrm{s}^{-1}$ \\
\hline
\end{tabular}

Note.

${ }^{a}$ Molecular gas associated with the star-forming region shown in blue in Figure 3.

\subsection{HST WFC3: PSF Subtraction}

We obtain archival Hubble Space Telescope (HST) observations of 3C 298 to quantify properties of the stellar populations of the host galaxy (GO13023; P.I. Chiaberge). Detailed descriptions of the observations are available in Hilbert et al. (2016). In summary, observations were taken in the F606W $\left(\lambda_{p}=588.7 \mathrm{~nm}, \quad\right.$ width $\left.=218.2 \mathrm{~nm}\right)$ and $\mathrm{F} 140 \mathrm{~W}$ $\left(\lambda_{p}=1392.3 \mathrm{~nm}\right.$, width $\left.=384.0 \mathrm{~nm}\right)$ filters, which cover the rest-frame wavelength range of $196.6 \mathrm{~nm}-286.1 \mathrm{~nm}$ and
$492.1-649.6 \mathrm{~nm}$, respectively. These wavelength ranges bracket the $4000 \AA$ break feature in galaxy spectra. Two observations were taken in each filter for a total exposure time of $1100.0 \mathrm{~s}$ (F606W) and $498.46 \mathrm{~s}$ (F140W).

We use the nearby star SDSS J141908.18+062834.7 to construct a point-spread function (PSF) for the F606W filter. The PSF star and quasar have a similar magnitude in the F606W filter with a similar $g-r$ color as measured in SDSS. Both the PSF star and quasar are saturated, so they share similar bleeding and diffraction patterns on the detector. In the F140W filter, the quasar is unsaturated and is about 0.8 magnitudes brighter, so we combine two unsaturated stars in the field to produce a final PSF with a matching $\mathrm{S} / \mathrm{N}$ in the diffraction spikes structure. We extract a $12^{\prime \prime} \times 12^{\prime \prime}$ box centered on the quasar and the PSF, and scale the flux of the PSF image to match the peak of the quasar emission and then subtract the two images. We also tried to match only the flux in the diffraction spikes that did not overlap with the structure in the host galaxy, and obtained a similar scaling factor. In both filters the inner $1^{\prime \prime}$ is dominated by noise from the PSF subtraction, the diffraction spikes at position angles of $0^{\circ}, 180^{\circ}$, $225^{\circ}$ and along the bleeding pattern in the F606W filter that extends about 0 " 5 along PA $0^{\circ}$ from the quasar. However, the majority of the host galaxy lies between position angles of $45^{\circ}$ and $170^{\circ}$, where the structure is least affected by residual noise. The quoted residual structure is for an image at a PA of $103^{\circ}$, matching the observations of OSIRIS and ALMA.

We convert electron counts at each pixel into flux density (Jy) using the 'PHOTFNU' header value. We resize the pixels to 100 mas plate scale to match OSIRIS and ALMA data by using the flux-conserving IDL frebin routine. We convert the maps into $\mathrm{AB}$ magnitude/ $\operatorname{arcsec}^{2}$ and construct a color map of the host galaxy. Reliable colors are extracted down to a surface brightness limit of $23.5 \mathrm{AB} \mathrm{mag} / \mathrm{arcsecond}^{2}$ for the majority of the host galaxy, with the exception of the redshifted outflow region that falls in the area dominated by residual noise from PSF subtraction.

Figure 10 presents the WFC3 F606W (left) and F140W (middle) after PSF subtraction in 0"' 1/pixel scale. The right panel shows the resolved host galaxy colors (F606W-F140W). Overlaying $\mathrm{H} \alpha$ contours on the color map shows that the starforming regions identified in OSIRIS (blue region in Figure 3) are nicely aligned with regions with bluer colors. Additionally, we overlay the ALMA CO (3-2) observation. The molecular clump offset by $16 \mathrm{kpc}$ from the quasar matches bluer regions where clumpy structure is seen in the F606W observations. This is evidence for young stellar populations in these regions, as would be expected from on-going star formation.

\subsection{HST WFC3: Stellar Population}

Two available filters for the 3C 298 system are ideally placed to bracket the break feature at a rest-frame wavelength of $4000 \AA$ found in galaxy spectra. Thus we can easily identify young $(<100 \mathrm{Myr})$ stellar populations without any ambiguity, as we attribute blue colors $($ F606W-F140W $<1.5)$ in these filters to stellar populations dominated by young $\mathrm{O}$ and $\mathrm{B}$ stars. Redder colors in these filters can come from older stellar populations (100 Myr-1 Gyr) or from young dusty star-forming regions, and thus we are unable to say much about older stellar populations without additional filters.

HST colors provide strong constraints on the resolved star formation and stellar population history across the 3C 298 

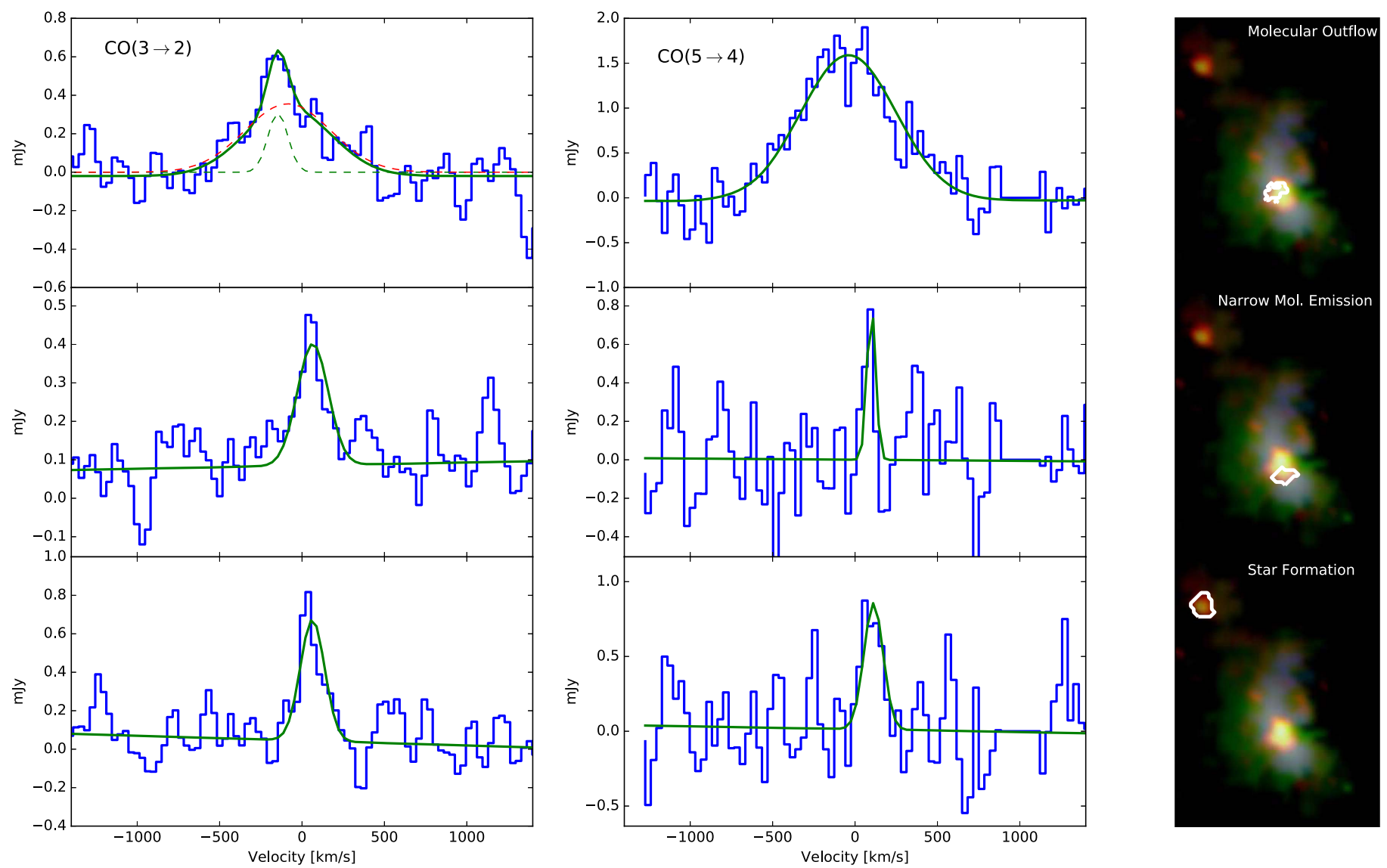

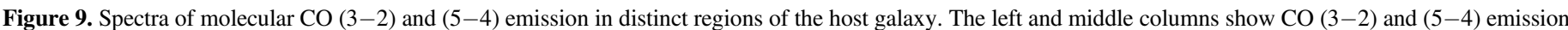

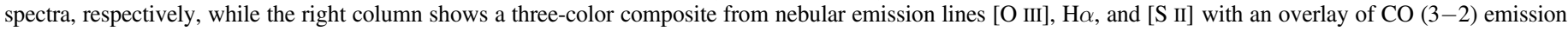

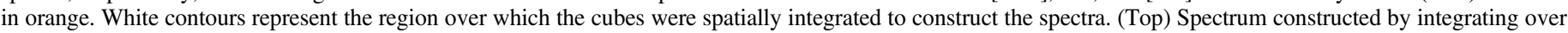

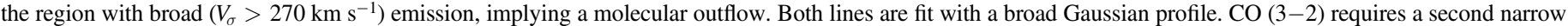

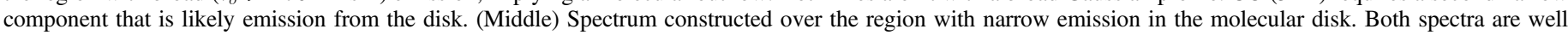

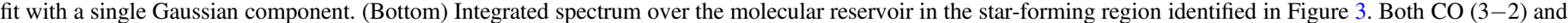
(5-4) spectra are fit with a narrow component.

system. We approximate the HST color map with the flexible stellar population synthesis (FSPS) code (Conroy et al. 2009; Conroy \& Gunn 2010), using a five-parameter star formation history model. We start the FSPS code with an exponential star formation history that is followed by a burst of star formation 2 Gyr later, using a solar metallicity and Salpeter IMF. Using the FSPS code, we find that varying the star formation history and e-folding the timescale $\tau$ from 0.1 to $10^{2}$ before the starburst does not significantly affect the age of the stellar population after the starburst.

The average F606W-F140W color over the blueshifted outflow region is best explained with a stellar population that has an age of $25 \mathrm{Myr}$ after the burst. According to ionized gas emission, dust does not affect the age estimated in this region since the $\mathrm{H} \alpha$ and $\mathrm{H} \beta$ ratio suggests a low $E(B-V) \sim 0$ value. However, ALMA observations reveal that part of the molecular disk centered on the quasar has extended emission into the blueshifted outflow region, suggesting that a part of the region can have higher dust extinction. The age of the outflow region and the modeled rest-frame U-V color imply that the stellar population resides in the transition zone between the red sequence and the blue cloud in the $\mathrm{U}-\mathrm{V}$ versus $M_{V}$ color diagram (Bell et al. 2004; Sánchez et al. 2004; Hopkins et al. 2008). Furthermore, $u-r$ modeled colors at this age suggest that the region lies where post-starburst galaxies reside in the $u-r$ versus $M_{\text {stellar }}$ diagram (Wong et al. 2012). The extracted 3C 298 model spectra shows strong Balmer absorption lines with a strong $4000 \AA$ A break, characteristic of a post-starburst galaxy. Stellar colors over the blueshifted outflow region are consistent with a strong episode of star formation that was abruptly halted.

In the region where star formation is identified in Figure 3 based on BPT diagnostics and in the molecular gas clump found in $\mathrm{CO}$, using the same initial conditions in the FSPS code with the outflow region, we find a stellar population with an age of $20 \mathrm{Myr}$ after a burst of star formation. Without any dust correction, this age should be taken as an upper limit. Ahead of the jet/blueshifted outflow, we find an even younger stellar population. From the $\mathrm{H} \alpha$ emission, we can place a limit of $0.3 M_{\odot} \mathrm{yr}^{-1} \mathrm{kpc}^{-2}$ across this region. The ALMA observation of the $\mathrm{CO}(3-2)$ transition yields a limit of $1 \times 10^{9} M_{\odot}$ on the molecular gas. This suggests that the current star formation rate is relatively low. However, the blue colors indicate recent star formation activity. The stellar populations in this region would then be associated with a young age of $7 \mathrm{Myr}$ after the burst. It is likely that the star formation event was very recent and short (6-10 Myr), which we deduce from the lack of $\mathrm{H} \alpha$ emission that would otherwise be present if star formation persisted for a time longer than $6 \mathrm{Myr}$.

Ages quoted in this analysis could be altered by additional quasar emission from light scattered off dust grains or electrons 


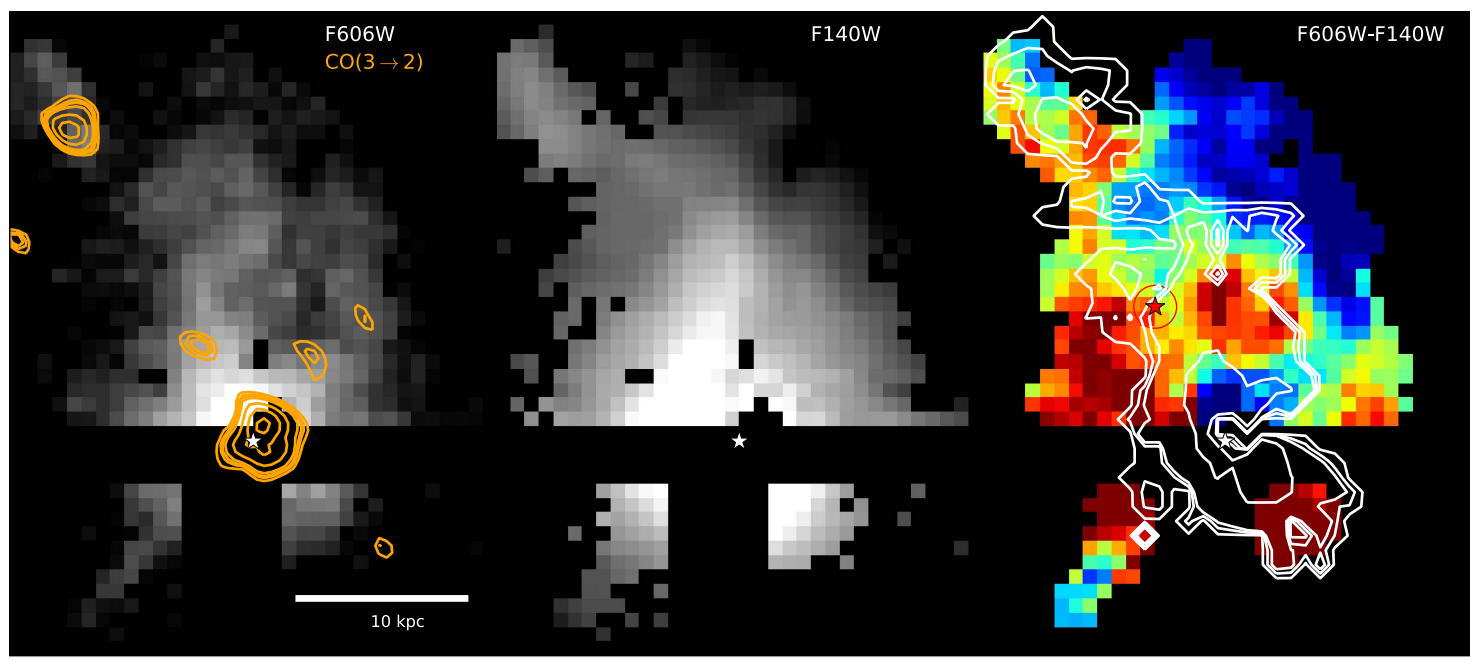

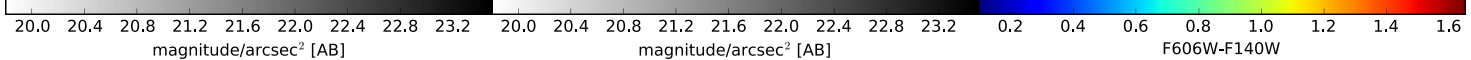

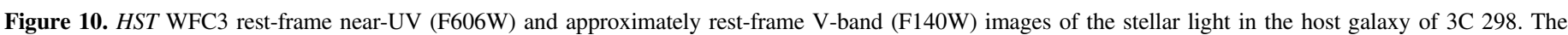

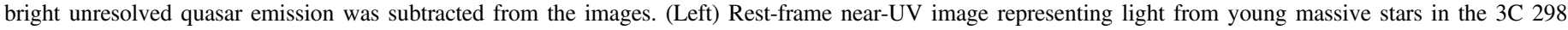

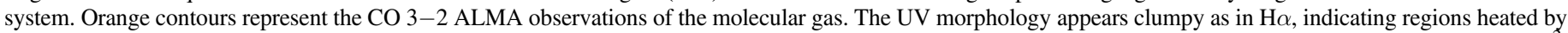

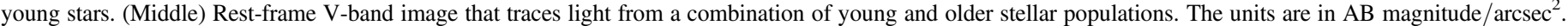

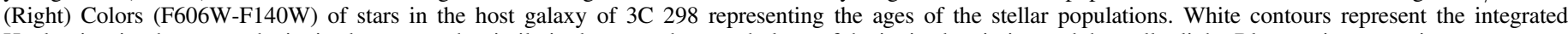

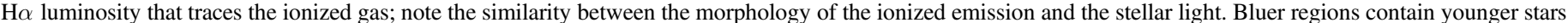

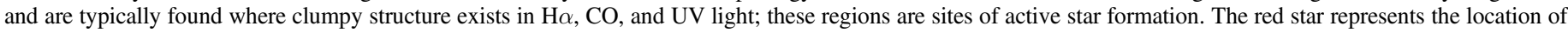
the dynamical center of the second merging galaxy in the system.

in the ISM, making the colors systemically bluer. However, polarimetric observations of 3C 298 reveal that the source is not heavily polarized, with an optical polarization of only $0.77 \pm 0.39 \%$ (Stockman et al. 1984). Given that the bluest regions also show star formation from other indicators (e.g., $\mathrm{H} \alpha$ and $\mathrm{CO}(3-2)$ emission), this implies that the majority of UV emission comes from young massive stars and not from scattered quasar light. The young stellar population observed $>16 \mathrm{kpc}$ beyond the blueshifted outflow is even farther away than the region that is photoionized by the quasar, indicating that ionizing radiation from the quasar does not reach this region.

\section{Dynamics and Energetics of Outflow Regions}

In this section we explore the dynamics and energetics of the ionized and molecular outflows identified in Sections 3.3, 3.4, and 3.7. We explore the energy budget from a broad absorption line (BAL) wind and from the quasar jets to investigate the origin of the galactic-scale winds.

\subsection{Outflow Rates}

The redshifted and blueshifted outflow regions resemble a cone-like structure. For a conical outflow with constant density $(\bar{\rho})$, we use the outflow rate equation from Cano-Díaz et al. (2012),

$$
\dot{M}=v R^{2} \Omega \bar{\rho},
$$

where $v$ is the velocity of the material in the outflow that is assumed to be moving at a constant rate over the cone, $R$ is the radial extent of the cone, and $\Omega$ is the opening angle of the cone. For $\bar{\rho}=M / V=\frac{3 M}{R^{3} \Omega}$, the outflow rate equation simplifies to

$$
\dot{M}=3 \frac{M v}{R} .
$$

When we assume that individual ionizing clouds in the outflow have the same density, we can derive an ionized gas mass from the line-integrated $\mathrm{H} \alpha$ luminosity and the average electron density over the outflow using (Osterbrock \& Ferland 2006)

$$
M_{\text {gas ionized }}=0.98 \times 10^{9}\left(\frac{L_{\mathrm{H} \alpha}}{10^{43} \mathrm{erg} \mathrm{s}^{-1}}\right)\left(\frac{n_{\mathrm{e}}}{100 \mathrm{~cm}^{-3}}\right)^{-1} .
$$

The electron density is derived from the line ratio of the [S II] lines in the blueshifted outflow region using a Gaussian fit to each component of the doublet. We find a ratio of ([S II] $6717 /[\mathrm{S}$ II] $6731=1.14)$, which yields an electron density of $272 \mathrm{~cm}^{-3}$ using the getTempDen code part of PyNeb (Luridiana et al. 2015) package. We calculate the ionized gas mass from the [O III] line using the method presented in CanoDíaz et al. (2012). This method requires knowledge of the ionized gas-phase metallicity at the site of the outflow region. We assume a metallicity value set to solar for a conservative estimate (i.e., for less than solar, the gas mass increases). For the outflow velocity we use the $\mathrm{v}_{10}$ parameter, which is the velocity value where $10 \%$ of the line is integrated. This value is calculated from the model fits to the spatially integrated [O III] and $\mathrm{H} \alpha$ lines for each outflow region. The wings of the emission lines most likely give the true average velocity of the outflow, as the lower velocities seen in the line profiles are probably due to projection effects of the conical structure (Cano-Díaz et al. 2012; Greene et al. 2012). The measured properties of the the outflow regions are presented in Table 3.

For the redshited and blueshifted outflow regions, we obtain a combined outflow rate of $467 M_{\odot} \mathrm{yr}^{-1}$ and $1515 M_{\odot} \mathrm{yr}^{-1}$ for 
Table 3

3C 298 Ionized Outflow Properties

\begin{tabular}{|c|c|c|c|c|c|c|c|c|}
\hline Region & $\begin{array}{c}L_{\mathrm{H} \alpha} \\
\times 10^{44} \mathrm{erg} \mathrm{s}^{-1} \\
\end{array}$ & $\begin{array}{c}L_{[\mathrm{O} \text { III }]} \\
\times 10^{44} \mathrm{erg} \mathrm{s}^{-1} \\
\end{array}$ & $\begin{array}{c}M_{\mathrm{H} \alpha} \\
\times 10^{8} M_{\odot} \\
\end{array}$ & $\begin{array}{c}M_{[\mathrm{O} \mathrm{III}]} \\
\times 10^{8} M_{\odot} \\
\end{array}$ & $\begin{array}{c}v \\
\mathrm{~km} \mathrm{~s}^{-1}\end{array}$ & $\begin{array}{c}R \\
\mathrm{kpc}\end{array}$ & $\begin{array}{c}\dot{M}_{\mathrm{H} \alpha} \\
M_{\odot} \mathrm{yr}^{-1}\end{array}$ & $\begin{array}{c}\dot{M}_{[\mathrm{O} \text { III }]} \\
M_{\odot} \mathrm{yr}^{-1}\end{array}$ \\
\hline Outflow-R & 0.29 & 1.8 & 10 & 3.5 & 1703 & 4.7 & 1110 & 390 \\
\hline Outflow-B & 0.09 & 0.3 & 3.2 & 0.6 & 1403 & 3.4 & 405 & 77 \\
\hline Outflow-AGN & 0.03 & 0.04 & 0.8 & 0.064 & 1400 & 3 & 115 & 9 \\
\hline
\end{tabular}

[O III] and $\mathrm{H} \alpha$, respectively, while for the AGN outflow region, we obtain an outflow rate of $9 M_{\odot} \mathrm{yr}^{-1}$ and $115 M_{\odot} \mathrm{yr}^{-1}$ for [O III] and $\mathrm{H} \alpha$. The $\mathrm{H} \alpha$ outflow rate is likely a better representation of the ionized outflow rate, since $\mathrm{H} \alpha$ is capable of probing denser regions where [O III] would be collisionally deexcited. The observed $\mathrm{H} \alpha$ outflow rate is similar to what is observed in other quasar surveys (Carniani et al. 2015), where a higher outflow rate is measured in $\mathrm{H} \alpha$ than in [O III]. The total kinetic energy and luminosity of the ionized outflow are $3.5 \times 10^{58} \mathrm{erg}$ and $1.2 \times 10^{45} \mathrm{erg} \mathrm{s}^{-1}$. The kinetic luminosity is consistent with the value usually invoked in theoretical models (Hopkins \& Elvis 2010) as necessary to affect starforming properties in the host galaxy. Over the outflow regions, we measure a $2 \sigma$ star formation rate upper limit of $0.3 M_{\odot} \mathrm{yr}^{-1} \mathrm{kpc}^{-2}$, by using a Gaussian profile with $2 \sigma$ peak flux and a FWHM of $200 \mathrm{~km} \mathrm{~s}^{-1}$, with the multi-Gaussian fit of the outflowing gas subtracted. The properties of the multiple outflow components are summarized in Table 3.

Similarly, using Equation (8), we compute the molecular outflow rate. As found in Section 3.7, the extent of the broad molecular emission from $\mathrm{CO}(3-2)$ and (5-4) lines is approximately $1.6 \mathrm{kpc}$, and the total molecular gas mass in the outflow region is $3.3 \times 10^{9} M_{\odot}$ with an outflow velocity of $400 \mathrm{~km} \mathrm{~s}^{-1}$ as measured by $V_{10}$ parameter. This yields a total molecular outflow rate of $2300 M_{\odot} \mathrm{yr}^{-1}$. The kinetic energy and luminosity of the molecular outflow are $5.1 \times 10^{57} \mathrm{erg}$ and $1 \times 10^{44} \mathrm{erg} \mathrm{s}^{-1}$.

\subsection{Virial Parameters and Gas Pressure}

Using the virial parameter, the ratio of the free-fall timescale to the dynamical timescale, we can investigate whether the gas is gravitationally bound or unbound in the outflow regions and molecular disk. A value of unity or lower would suggest that the gas is bound and is able to collapse to form stars. A value much greater than one would suggest that the gas is unbound and therefore at the present time should not be collapsing to form stars. Over the outflow region, we measure a virial parameter

$$
\alpha_{\mathrm{vir}}=5 \frac{\sigma^{2} R}{G M} \approx 164\left(\frac{\sigma}{500 \mathrm{~km} \mathrm{~s}^{-1}}\right)^{2},
$$

using an average velocity dispersion of $\sigma=500 \mathrm{~km} \mathrm{~s}^{-1}$ seen in the ionized outflow, a radius of $R=3 \mathrm{kpc}$, and the ionized gas mass $\left(1.32 \times 10^{9} M_{\odot}\right)$. Using the molecular gas mass of $6.6 \times 10^{9} M_{\odot}$, an average velocity dispersion of $270 \mathrm{~km} \mathrm{~s}^{-1}$ seen in the molecular outflow and a radius of $1.6 \mathrm{kpc}$ yields $\alpha_{\mathrm{vir}}$ of 20.5 . Both of these virial parameter estimates suggest that at the present time, the physical conditions in the ionized and molecular outflow regions and in the molecular disk are stable against gravitational collapse, hindering star formation. In contrast, the molecular gas clump in the star-forming region observed in CO (3-2) yields a virial parameter value of $\alpha_{\mathrm{vir}} \approx 0.7$. A value close to unity suggests that star formation should be able to proceed in this region, where there are no powerful outflows.

If the quasar-driven wind does not efficiently cool and adiabatically expands, then the initial driving mechanism is capable of transferring its kinetic energy into mechanical energy in the galactic-scale outflow. The momentum flux of the outflow should therefore be greater than $L_{\text {bol }} / \mathrm{c}$ (Zubovas \& King 2012). We measure a total momentum flux $\left(\dot{P}_{\text {outflow }}=\dot{M} \times v\right)$ of $2.1 \times 10^{37}$ dynes over the entire outflow region. We use the $3000 \AA$ quasar luminosity to compute a bolometric luminosity value of $1.04 \times 10^{47} \mathrm{erg} \mathrm{s}^{-1}$ (Runnoe et al. 2012). Comparing the outflow momentum flux to the quasar radiation momentum flux $\left(L_{\mathrm{bol}} / c\right)$ of $3.5 \times 10^{36}$ dynes yields a ratio $\dot{P}_{\text {outflow }} / \dot{P}_{\text {quasar }}$ (loading factor) of 6 . In principle, this ratio is a lower limit, since there can still be diffuse molecular gas in the ionized outflow regions whose $\mathrm{CO}$ emission is below the sensitivity of ALMA. Based on theoretical work by Faucher-Giguère et al. (2012) and Zubovas $\&$ King (2012), the measured loading factor of $>6$ suggests that the wind is energy conserving. The total kinetic luminosity of the outflow is also about $2 \%$ of $\mathrm{L}_{\text {bol }}$, consistent with the above theoretical prediction. The observed extended outflow properties of 3C 298 strongly suggest that radiation pressure by itself is unable to drive the outflow. Star formation is also incapable of driving the observed outflow because the expected terminal velocity for a supernova-driven galactic-scale wind is only $\sigma \sim 200 \mathrm{~km} \mathrm{~s}^{-1}$ (Murray et al. 2005), far lower than the observed velocities $\left(\sigma \sim 500 \mathrm{~km} \mathrm{~s}^{-1}\right)$ seen in the biconical wind.

The outflow could be induced by either the jet and/or BAL winds from the quasar. We infer a jet pressure

$$
P_{\mathrm{jet}} \approx \frac{L_{\mathrm{jet}} \times t}{3 \mathrm{~V}}
$$

of $3 \times 10^{-8}$ dynes $\mathrm{cm}^{-2}$ using the jet kinetic luminosity of $1.4 \times 10^{47} \mathrm{erg} \mathrm{s}^{-1}$, derived using the $1.4 \mathrm{GHz}(570 \mathrm{MHz}$ observed) radio flux-jet power relation (Bîrzan et al. 2008; Cavagnolo et al. 2010), assuming spherical volume at a radius of $9 \mathrm{kpc}$ and a timescale of $3 \mathrm{Myr}$. This is an order-ofmagnitude calculation given the approximations in the jet luminosity, the lifetime (t) of the jet, and the volume. Observations of other BAL quasars indicate that the kinetic luminosity of outflows can become as high as $10^{46} \mathrm{erg} \mathrm{s}^{-1}$ (Chamberlain et al. 2015), which is close to the kinetic luminosity for the 3C 298 jet and would exert a pressure of $10^{-8}$ dynes $\mathrm{cm}^{-2}$. This means that a BAL-type wind could potentially drive the extended 3C 298 outflow. However, there is no observed BAL in the optical and infrared spectra of $3 \mathrm{C}$ 298. The lack of an observed BAL in 3C 298 could be due to an orientation effect where our line of sight does not overlap with the absorbing outflowing gas. If this is not the case and 
there is no BAL, the jet of 3C 298 still has the necessary ram pressure capable of inducing a large-scale outflow.

The gas pressure $(P=n k T)$ in the ionized ([S II]) gas is $\sim 4 \times 10^{-10}$ dynes $\mathrm{cm}^{-2}$. From the ALMA CO (3-2) observations, we measure a molecular gas surface density of $490 M_{\odot} \mathrm{pc}^{-1}$, indicating a molecular gas pressure $\left(P=(\pi / 2) G \Sigma_{\text {molecular }}^{2}\right)$ of $\sim 1 \times 10^{-9}$ dynes $\mathrm{cm}^{-2}$. Both the BAL wind and quasar jet pressures are 2-3 orders of magnitude higher than the current pressure of the ionized and molecular ISM. The fact that the ionized gas pressure is comparable to or lower than the weight per unit area of the molecular gas shows that the jet is not currently producing an overpressure (which would be reflected in the thermal gas pressure of the ionized gas in the ISM) relative to the overburden of the molecular gas. This suggests that the jet is now venting out of the galaxy. The interaction between the ISM and the jet and/or BAL wind in the past was likely in a denser environment, and thus confined to a smaller volume with a higher pressure, to initially generate the outflows.

Both a BAL quasar wind and jet have enough kinetic energy to drive galactic-scale winds. These large outflows in 3C 298 supply the necessary energy and momentum to hinder star formation along their path and expel large amounts of gas from the galaxy. If the sources of turbulence were to halt at the present time, the shocks would dissipate on a dynamical timescale of 3-6 Myr. By that time, the majority of the gas in the ionized outflow will have already escaped into the intergalactic medium (IGM), with most of the molecular gas in the disk removed from the inner few kiloparsec and potentially swept up into the ionized outflow. Any left-over gas in the galaxy would quickly cool back into a molecular state. Taken together, this shows both negative and ejective feedback occurring along the outflow region, thereby impacting the stellar mass growth of the quasar host.

\section{Discussion}

To generate a comprehensive picture of 3C 298, we combine multiwavelength data to spatially resolve the ionized gas, molecular gas, and stellar light distribution of the host galaxy. This allows a unique comparison between the star formation histories, photoionization mechanisms, and gas-phase properties in a high-redshift quasar host galaxy. In Figure 11 we generalize the multiwavelength analysis of the 3C 298 system in a schematic diagram and summarize its properties in Table 4.

We make use of observations from the VLA to probe the quasar radio jets, WFC3 data from the $H S T$ to study the stellar contribution, from ALMA to measure the resolved molecular gas morphology and dynamics, and from the Keck LGS-AO to determine the resolved ionized gas properties. These data show clear evidence of a conical outflow in the host galaxy of $3 \mathrm{C}$ 298, driven by quasar jets and/or winds, that directly impact the ISM of the host galaxy. VLA images of 3C 298 reveal synchrotron radio jets feeding lobes that extend over $\sim 18 \mathrm{kpc}$ from the quasar in the east-west direction (Figure 1). Along the radio lobes, OSIRIS kinematic maps reveal broad $\left(\sigma \sim 800 \mathrm{~km} \mathrm{~s}^{-1}\right)$ emission lines that are offset by up to $600 \mathrm{~km} \mathrm{~s}^{-1}$ from the quasar's systemic redshift, indicating an outflow extending over a significant swathe of the galaxy.

We find direct evidence of negative feedback along the path of the jet and outflow regions of the host galaxy. The term "negative feedback" has been used loosely in the astronomical literature; here we define negative feedback as injected energy

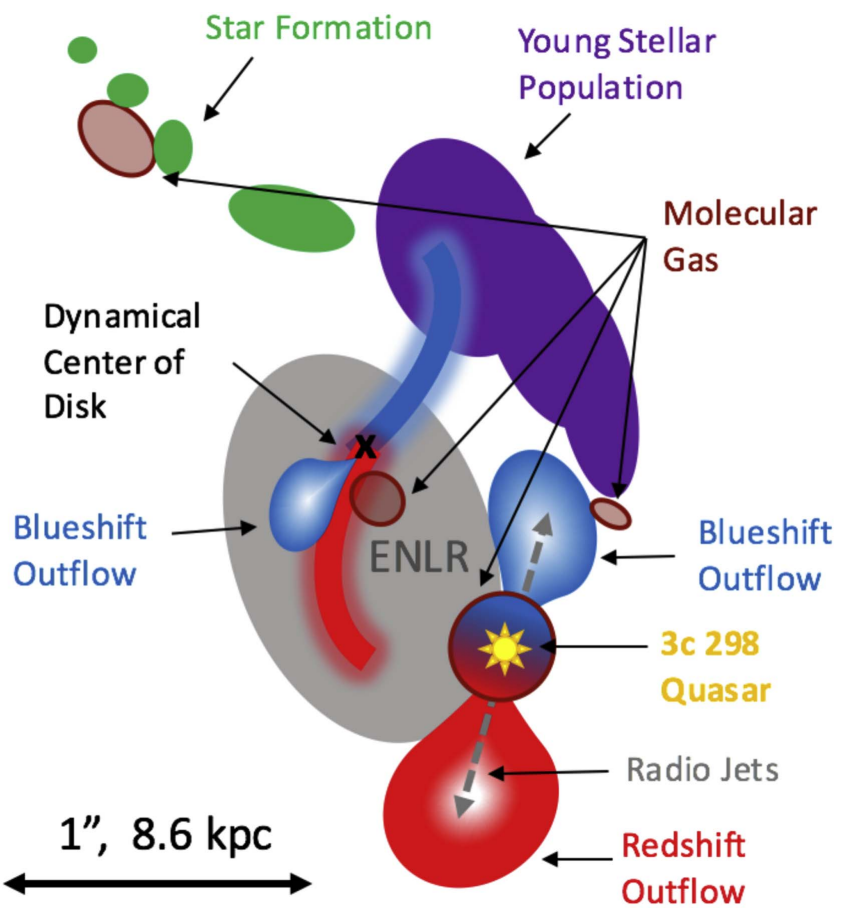

Figure 11. Cartoon illustration of the merger in the 3C 298 system that includes the results from OSIRIS, ALMA, $H S T$, and VLA. The location of the quasar is highlighted with a yellow star. The large blue- and redshifted outflows emanating from the quasar observed in the nebular emission lines are coaligned with the radio jet (indicated by a gray dashed arrow), where negative feedback is observed. The locations of the ALMA CO (3-2) and (5-4) emission are shown with red-brown ovals, representing molecular gas reservoirs. A molecular disk centered on the quasar is shown with a blue-red gradient. We find strong evidence for molecular outflow on the blueshifted side of the disk. The dynamical center of the secondary merging galaxy is shown with a black cross. The radial-velocity map of this galaxy fits the disk model well. A secondary outflow region is found to lie near the dynamical center of the merging galaxy, providing tentative evidence for a secondary AGN in the system. The secondary AGN has spatially concentrated elevated emission-line ratios that are consistent with ionization from an AGN. A large area of the $3 \mathrm{C}$ 298 system is observed to have an ENLR (gray) between the quasar and putative AGN. The majority of the star formation (green) occurs in compact clumps that are offset from the two galaxies, which would be consistent with a tidal feature being induced by the merger. A young stellar population (purple) measured from the HST WFC 3 colors is offset from both the outflows and the molecular gas of the system.

and momentum in the ISM that inhibits the normal thermal cooling and/or decay of turbulence, thereby extending the timescale for star formation to occur. Galactic-scale feedback may also occur when high-velocity outflows remove large fractions of the ISM, thereby impacting the star formation and stellar mass history of the host galaxy. The latter might be called "ejective feedback." Both negative and ejective feedback may occur simultaneously. The 3C 298 ionized outflow region has a biconical shape with a primarily blueshifted approaching side and redshifted receding side of the cone (Figures 1 and 3). Emission-line ratios (e.g., $[\mathrm{N} \mathrm{II}] / \mathrm{H} \alpha$ and $[\mathrm{O} \mathrm{III]} / \mathrm{H} \beta$ ) allow us to distinguish between various photoionization modes of the ISM of 3C 298, which is illustrated in the nebular diagnostic diagram in Figure 3. In both of the outflow regions, we detect high nebular emission-line ratios that imply a combination of hard photoionizing radiation from the quasar and shock ionization. We find a total ionized mass outflow rate $(\dot{M})$ of 450-1500 $M_{\odot} \mathrm{yr}^{-1}$ combined for both outflow regions. ALMA observations of molecular gas reveal a disk centered on the quasar. A large fraction of gas in the molecular disk reveals 
Table 4

3C 298 Properties and Results

\begin{tabular}{|c|c|}
\hline Parameter & Value \\
\hline$z_{\text {quasar }}$ & 1.439 \\
\hline R.A. & 14:19:08.181 \\
\hline Decl. & $+06: 28: 34.79$ \\
\hline$\lambda L_{\lambda}^{\mathrm{a}}$ & $2.0 \times 10^{46} \mathrm{erg} \mathrm{s}^{-1}$ \\
\hline SFR H $\alpha$ & $88 \pm 9 M_{\odot} \mathrm{yr}^{-1}$ \\
\hline SFR IR ${ }^{b}$ & $930_{-40}^{+40} M_{\odot} \mathrm{yr}^{-1}$ \\
\hline$M_{\text {dust }}^{\mathrm{b}}$ & $3.8_{-0.4}^{+0.3} \times 10^{8} M_{\odot}$ \\
\hline$M_{\mathrm{H}_{2}}^{\text {Total }}$ & $8.75 \pm 0.4 \times 10^{9} M_{\odot}$ \\
\hline$M_{\mathrm{H}_{2}}^{\mathrm{Mol} \text { Disc }}$ & $6.6 \pm 0.36 \times 10^{9} M_{\odot}$ \\
\hline$M_{\mathrm{H}_{2}}^{\mathrm{SF} \text { Region }}$ & $1.4 \pm 0.14 \times 10^{9} M_{\odot}$ \\
\hline$\dot{M}_{[\mathrm{O} \text { III }]}$ & $467 M_{\odot} \mathrm{yr}^{-1}$ \\
\hline$\dot{M}_{\mathrm{H} \alpha}$ & $1515 M_{\odot} \mathrm{yr}^{-1}$ \\
\hline$\dot{M}_{\mathrm{H}_{2}}$ & $2300 M_{\odot} \mathrm{yr}^{-1}$ \\
\hline Outflow $n_{\mathrm{e}}{ }^{\mathrm{c}}$ & $272 \mathrm{~cm}^{-3}$ \\
\hline$M_{\text {dyn, bulge }}$ & $1.35 \pm 0.5 \times 10^{10}$ \\
\hline$M_{\mathrm{SMBH}}$ & $3.23 \pm 1.1 \times 10^{9}$ \\
\hline
\end{tabular}

Notes.

a Computed at rest-frame $3000 \AA$.

${ }^{\mathrm{b}}$ Value from Podigachoski et al. (2015).

${ }^{\mathrm{c}}$ Value based on the [S II] line ratio measurment over the blueshifted outflow region.

broad emission associated with a molecular outflow with a rate of $2300 M_{\odot} \mathrm{yr}^{-1}$. Unlike the ionized outflow, the molecular outflow emanates in a single direction from the blueshifted side of the molecular disk. The molecular outflow either originates from the 3C 298 quasar nuclear region or from the disk itself. In the rest-frame of the redshifted ionized receding cone, two broad Gaussian functions are required to fit the profiles of the nebular emission lines (Figure 2). This indicates an approaching and receding component, corresponding to the front and back of a hollow cone that suggests expansion of the outflow along our line of sight.

Combining kinematic maps from OSIRIS and ALMA reveals that the ionized outflow gas most likely originates from the molecular disk since the blue-/redshifted outflow matches the blue-/redshifted region of the disk. Furthermore, the blueshifted molecular outflow is on the same side as the ionized. We find no narrow $\left(\sigma<200 \mathrm{~km} \mathrm{~s}^{-1}\right) \mathrm{H} \alpha$ emission in either of the outflow regions, suggesting that the majority of the ionized gas is in a turbulent phase. The outflow carries a significant amount of kinetic energy, and it is capable of removing the majority of the galaxy gas on a timescale of 3 Myr. The virial parameter deduced from the ionized and molecular gas over the outflow regions is much greater than one, implying that the ISM is not self-gravitating, and hence will not collapse to form stars. Similarly, the molecular outflow is both stirring the molecular gas and removing it from the inner regions of the galaxy. Ionization occurring over the outflow region is dominated by shocks, which drives intense heating into the ISM that further inhibits star formation. Over the conical outflow region, we derive a conservative limit for $\mathrm{H} \alpha$ emission due to star formation of $<14 M_{\odot} \mathrm{yr}^{-1}(2 \sigma)$, or $\sim 0.3 M_{\odot} \mathrm{yr}^{-1} \mathrm{kpc}^{-2}$ using the empirical $\mathrm{H} \alpha$ luminosity-star formation rate (Kennicutt 1998). Colors of the stellar light as seen in HST data projected on the blueshifted outflow region are also consistent with a stellar population whose star formation was abruptly halted within the last $\sim 25 \mathrm{Myr}$ (see Figure 10).

In the 3C 298 host galaxy we observe star-forming regions that are offset from the outflows. The youngest ( 7 Myr) stellar populations observed with the HST (Figure 10) imply that a recent burst of star formation has occurred. These regions are found $10-16 \mathrm{kpc}$ east of the quasar. In addition, beyond the extended narrow-line and outflow regions, using OSIRIS and the $H S T$, we resolve star-forming clumps that lie $\sim 1-22 \mathrm{kpc}$ (blue in Figure 3) away from the quasar. These clumps have an integrated star formation rate of $88 \pm 9 M_{\odot} \mathrm{yr}^{-1}$, based on the observed $\mathrm{H} \alpha$ luminosity and empirical star formation rate relation (Kennicutt 1998). ALMA CO (3-2) and (5-4) data reveal a molecular clump in the vicinity of this region with a total molecular gas mass of $1.4 \times 10^{9} M_{\odot}$. Using the HSTmeasured colors, we find a young stellar population with an age of $20 \mathrm{Myr}$ in these star-forming regions. Therefore, combining results from the resolved molecular gas, stellar populations, and ionized gas yields a consistent picture; we find star formation occurring away from large-scale outflows, where the quasar winds and/or jets are stirring the ISM and removing gas.

The kinematic map of the ionized gas in 3C 298 shows a systematic velocity gradient across a large portion of the system. We fit this region with a rotating-disk model (Figure 5). We find evidence for a second rotating disk in the system that is offset by $\sim 8.6 \mathrm{kpc}$ and $\sim 170 \mathrm{~km} \mathrm{~s}^{-1}$ from the quasar centroid and central velocity of the broad-line region. The dynamical center of the second disk is in the vicinity of a secondary outflow region, suggesting the presence of a candidate second nucleus. The offset between the dynamical center of the second disk and the secondary outflow is on the order of $3 \mathrm{kpc}$. We note that this is a typical separation that is found between the stellar centroid and AGN location in local late-stage mergers (Liu et al. 2013b; Comerford et al. 2015; Barrows et al. 2016). We infer that the 3C 298 system likely has two massive galaxies with distinct rotating disks that are currently experiencing a close passage, where their galactic nuclei and SMBHs have yet to merge.

We calculated the enclosed dynamical mass of the molecular disk at the location of the quasar to be $1-1.7 \times 10^{10} M_{\odot}$. With the measured SMBH mass $\left(10^{9.37-9.56} M_{\odot}\right)$, 3C 298 resides off the local scaling $M_{\text {bulge }}-M_{\mathrm{BH}}$ relationship, which indicates that black hole growth must occur earlier than stellar mass assembly. These results are similar to what has recently been observed in nearby ULIRGs in gas-rich merger stages by Medling et al. (2015), where they find that these ULIRGs predominantly have early black hole growth and have yet to form the bulk of their stellar mass. Similar results have been found for a small number of $\mathrm{z}>6$ quasar host galaxies observed with ALMA, where the ratio of the black hole to the galaxy bulge mass is higher by a factor of 3-4 (Wang et al. 2013; Venemans et al. 2016) than in local galaxies. This contradicts previous theoretical simulations, which imply that star formation and stellar mass build-up should occur during the gas-rich merger phase, before the SMBH experiences its predominant growth when AGN feedback is assumed to transpire (i.e., Di Matteo et al. 2005; Hopkins 2012; Hopkins et al. 2016).

Models are still unclear about whether distant quasars should lie above, below, or even on an extension of the local SMBHgalaxy scaling relations. Hydrodynamical simulations of $z>6$ 
quasars by Barai et al. (2017) suggest that negative feedback may drive quasars above the local scaling SMBH-galaxy relations. Barai et al. (2017) argue that conical, symmetric outflows can remove gas efficiently from galactic disks while still allowing gas to accrete onto the SMBH perpendicular to the biconical outflow. Furthermore, the same simulations without quasar feedback tend to grow their stellar mass ahead of the SMBH, driving galaxies below the local correlations. Anglés-Alcázar et al. (2017) also find that the stellar bulges outpace the SMBH in the absence of feedback. Anglés-Alcázar et al. (2017) include feedback from bursty star formation, which can limit the growth of the SMBH and keeps the system at a location below the local SMBH-galaxy scaling relation.

The source 3C 298 at $z=1.439$ (age $4.47 \mathrm{Gyr}$ ) has quasar negative feedback occurring in a conical outflow early in the gas-rich merger phase, implying that the majority of the nucleus' stellar mass assembly must occur in later phases if it is to reach current scaling relations. Since the majority of the current gas supply in the host galaxy of 3C 298 is incapable of forming stars and will soon be removed from the galaxy through the quasar-driven winds, perhaps this assembly occurs through dry mergers or newly supplied IGM molecular gas. Even if all of the current gas supply in the molecular disk $\left(6.6 \times 10^{9} M_{\odot}\right)$ turns into stars, it will still be insufficient to bring the bulge of the 3C 298 host galaxy onto the local scaling relation. For the 3C 298 system, this means that the SMBH mass is formed earlier and more efficiently than the stellar mass of the host galaxy.

The host galaxy of 3C 298 could be a precursor to local galaxies that are significantly offset from the local scaling relation (e.g., NGC 1277). If there is insufficient accretion from the IGM to fuel future star formation or if 3C 298 resides in a less dense environment than a typical quasar, it could fail to form/accrete enough stars to fall on the local scaling laws. A natural way to limit the growth of the stellar bulge in a system with a powerful AGN is to have strong feedback on the ISM through a bipolar outflow early in the lifetime of the system (Fabian et al. 2013).

In summary, the 3C 298 system shows strong evidence of negative feedback from powerful conical outflows that are halting star formation by driving extremely high-velocity turbulence in the ISM and by ejecting large amounts of gas. The dynamical timescale of the outflow is $\sim 3-6 \mathrm{Myr}$, suggesting that quenching of star formation must occur rapidly. In the 3C 298 system, strong feedback has started fairly early in the merger process, well before the final coalescence of the two galactic nuclei. The system shows multiple concurrent phases of the standard merger model (Sanders et al. 1988; Hopkins et al. 2008; Alexander \& Hickox 2012), with the coexistence of a luminous type-1 quasar, star formation in merger host galaxies, and a candidate secondary AGN that is offset from the quasar. This implies that removal of gas and dust surrounding the SMBHs occurred rapidly and early during the merger phase. This early and short onset of quasar feedback compared to total timescale of the merger $(\sim 1 \mathrm{Gyr})$ may also partially explain why it has been difficult to measure its effect on the star-forming ISM in distant galaxies.

\section{Conclusion}

We presented integral field spectroscopy of the resolved nebular emission lines $\mathrm{H} \beta$, [O III], $\mathrm{H} \alpha$, [N II], and [S II]together with rotational $\mathrm{CO}(3-2)$ and $(5-4)$ spatially resolved emission spectroscopy in the host galaxy of the radio-loud quasar 3C $298(z=1.439)$. These data were supplemented with archival HST-WFC3 and VLA observations of the restframe UV and optical stellar continuum and jet/lobe synchrotron emission, respectively.

1. We find strong evidence for negative feedback in the host galaxy of 3C 298. Dynamics of ionized gas traced through nebular emission lines show a significant quasardriven outflow encompassing a large area of the host galaxy. We derive an ionized outflow rate of 467-1515 $M_{\odot} \mathrm{yr}^{-1}$.

2. Cospatial with the powerful ionized gas outflow, VLA imaging shows extended synchrotron emission from the quasar jet/lobes. Radio data suggest that the jet has sufficient energy to drive the outflow. A BAL wind also has the necessary energetics for potentially driving the outflow.

3. We detect a molecular gas disk centered on the quasar with a total molecular gas of $6.6 \times 10^{9} M_{\odot}$ and effective radius of $1.6 \mathrm{kpc}$.

4. A powerful quasar-driven molecular outflow is detected in the molecular disk with an $\dot{M}_{\mathrm{H}_{2}}$ outflow rate of $2300 M_{\odot} \mathrm{yr}^{-1}$. The molecular gas in the disk will be depleted by the outflow on a timescale of $3 \mathrm{Myr}$.

5. Dynamical modeling of the molecular disk reveals that total mass enclosed in the disk is 2-2.5 orders of magnitude below the expected value from the local $M_{\text {stellar-bulge }}-M_{\mathrm{BH}}$ relationship using the measured $\mathrm{SMBH}$ mass of the 3C 298 quasar.

6. Several kiloparsecs away from the outflow path, we find evidence of star-forming regions based on BPT diagnostics, strong UV emission from $\mathrm{O}$ and $\mathrm{B}$ stars, and evidence for a molecular reservoir with a total mass of $1.4 \times 10^{9} M_{\odot}$.

7. Disk modeling of the velocity field traced by [O III] emission shows a second rotating disk with a dynamical center offset from the quasar by $9 \mathrm{kpc}$. This suggests evidence for a late-stage merger where the disk traces the second merging galaxy in the system. The dynamical center of the disk aligns well with a secondary outflow region seen in nebular emission that does not extend from the quasar and does not align with any structure in the radio map. This suggests a candidate secondary AGN in the 3C 298 system.

8. These observations taken together imply an early onset of negative feedback with a short quenching time compared to the timescale of the galactic merger. Feedback also occurs early in the 3C 298 merger process, and it occurs well before the assembly of the 3C 298 host galaxy on the local $M_{\text {stellar-bulge }}-M_{\mathrm{BH}}$ relationship.

The authors wish to thank Randy Campbell and Jim Lyke for their assistance at the telescope in acquiring the Keck OSIRIS data sets. We also appreciate valuable discussions with Dusan Keres and the constructive comments made by the anonymous referee. The data presented herein were obtained at the W.M. Keck Observatory, which is operated as a scientific partnership among the California Institute of Technology, the University of California and the National Aeronautics and Space Administration. The Observatory was made possible by the generous 
financial support of the W.M. Keck Foundation. The authors wish to recognize and acknowledge the very significant cultural role and reverence that the summit of Maunakea has always had within the indigenous Hawaiian community. We are most fortunate to have the opportunity to conduct observations from this mountain. This paper makes use of the following ALMA data: ADS/JAO.ALMA[2013.1.01359.S]. ALMA is a partnership of ESO (representing its member states), NSF (USA), and NINS (Japan), together with NRC (Canada), NSC, and ASIAA (Taiwan), and KASI (Republic of Korea), in cooperation with the Republic of Chile. Based on observations made with the NASA/ESA Hubble Space Telescope, obtained from the Data Archive (Program GO13023) at the Space Telescope Science Institute, which is operated by the Association of Universities for Research in Astronomy, Inc., under NASA contract NAS 5-26555. A portion of the research was conducted at The Dunlap Institute for Astronomy and Astrophysics that is funded through an endowment established by the David Dunlap family and the University of Toronto. This research has made use of the NASA/IPAC Extragalactic Database (NED) which is operated by the Jet Propulsion Laboratory, California Institute of Technology, under contract with the National Aeronautics and Space Administration.

Facilities: Keck (OSIRIS-LGSAO), ALMA (Band 4 and 6), HST(WFC3), VLA.

Software: Scipy: (Jones et al. 2001), CASA: (McMullin et al. 2007), OSIRIS Data Reduction Pipeline: (Lyke et al. 2017), Matplotlib (Hunter 2007).

\section{ORCID iDs}

James E. Larkin (1) https://orcid.org/0000-0001-7687-3965

\section{References}

Alexander, D. M., \& Hickox, R. C. 2012, NewAR, 56, 93

Allen, M. G., Groves, B. A., Dopita, M. A., Sutherland, R. S., \& Kewley, L. J. 2008, ApJS, 178, 20

Anglés-Alcázar, D., Faucher-Giguère, C.-A., Quataert, E., et al. 2017, arXiv: 1707.03832

Barai, P., Gallerani, S., Pallottini, A., et al. 2017, arXiv:1707.03014

Barrows, R. S., Comerford, J. M., Greene, J. E., \& Pooley, D. 2016, ApJ, 829,37

Bell, E. F., McIntosh, D. H., Barden, M., et al. 2004, ApJL, 600, L11

Benson, A. J., Bower, R. G., Frenk, C. S., et al. 2003, ApJ, 599, 38

Bîrzan, L., McNamara, B. R., Nulsen, P. E. J., Carilli, C. L., \& Wise, M. W. 2008, ApJ, 686, 859

Bruce, V. A., Dunlop, J. S., McLure, R. J., et al. 2014, MNRAS, 444, 1660

Brusa, M., Perna, M., Cresci, G., et al. 2016, A\&A, 588, A58

Calzetti, D., Armus, L., Bohlin, R. C., et al. 2000, ApJ, 533, 682

Cano-Díaz, M., Maiolino, R., Marconi, A., et al. 2012, A\&A, 537, L8

Carilli, C. L., \& Walter, F. 2013, ARA\&A, 51, 105

Carniani, S., Marconi, A., Maiolino, R., et al. 2015, A\&A, 580, A102

Carniani, S., Marconi, A., Maiolino, R., et al. 2016, A\&A, 591, A28

Cavagnolo, K. W., McNamara, B. R., Nulsen, P. E. J., et al. 2010, ApJ, 720, 1066

Chamberlain, C., Arav, N., \& Benn, C. 2015, MNRAS, 450, 1085

Cicone, C., Maiolino, R., Sturm, E., et al. 2014, A\&A, 562, A21

Comerford, J. M., Pooley, D., Barrows, R. S., et al. 2015, ApJ, 806, 219

Conroy, C., \& Gunn, J. E. 2010, ApJ, 712, 833

Conroy, C., Gunn, J. E., \& White, M. 2009, ApJ, 699, 486

Costa, T., Sijacki, D., \& Haehnelt, M. G. 2015, MNRAS, 448, L30

Cresci, G., Mainieri, V., Brusa, M., et al. 2015, ApJ, 799, 82

Delvecchio, I., Gruppioni, C., Pozzi, F., et al. 2014, MNRAS, 439, 2736

Di Matteo, T., Springel, V., \& Hernquist, L. 2005, Natur, 433, 604

Fabian, A. C., Sanders, J. S., Haehnelt, M., Rees, M. J., \& Miller, J. M. 2013, MNRAS, 431, L38
Falomo, R., Kotilainen, J. K., Scarpa, R., \& Treves, A. 2005, A\&A, 434, 469 Fanti, C., Fanti, R., Dallacasa, D., et al. 2002, A\&A, 396, 801

Faucher-Giguère, C.-A., Quataert, E., \& Murray, N. 2012, MNRAS, 420, 1347 Ferrarese, L., \& Merritt, D. 2000, ApJL, 539, L9

Floyd, D. J. E., Dunlop, J. S., Kukula, M. J., et al. 2013, MNRAS, 429, 2 Gallazzi, A., Brinchmann, J., Charlot, S., \& White, S. D. M. 2008, MNRAS, 383,1439

Gebhardt, K., Bender, R., Bower, G., et al. 2000, ApJL, 539, L13

Glikman, E., Simmons, B., Mailly, M., et al. 2015, ApJ, 806, 218

Greene, J. E., Zakamska, N. L., \& Smith, P. S. 2012, ApJ, 746, 86

Häring, N., \& Rix, H.-W. 2004, ApJL, 604, L89

Harrison, C. M., Alexander, D. M., Mullaney, J. R., \& Swinbank, A. M. 2014, MNRAS, 441, 3306

Harrison, C. M., Alexander, D. M., Swinbank, A. M., et al. 2012, MNRAS, 426, 1073

Hilbert, B., Chiaberge, M., Kotyla, J. P., et al. 2016, ApJS, 225, 12

Holmberg, E. 1946, MeLuS, 117, 3

Hopkins, P. F. 2012, MNRAS, 420, L8

Hopkins, P. F., \& Elvis, M. 2010, MNRAS, 401, 7

Hopkins, P. F., Hernquist, L., Cox, T. J., \& Kereš, D. 2008, ApJS, 175, 356

Hopkins, P. F., Torrey, P., Faucher-Giguère, C.-A., Quataert, E., \& Murray, N. 2016, MNRAS, 458, 816

Hunter, J. D. 2007, CSE, 9, 90

Hutchings, J. B., Frenette, D., Hanisch, R., et al. 2002, AJ, 123, 2936

Inskip, K. J., Jahnke, K., Rix, H.-W., \& van de Ven, G. 2011, ApJ, 739, 90

Jahnke, K., Sánchez, S. F., Wisotzki, L., et al. 2004, ApJ, 614, 568

Jones, E., Oliphant, T., Peterson, P., et al. 2001, SciPy: Open Source Scientific Tools for Python, (Online; accessed 2016-11-22) http://www.scipy.org/

Kauffmann, G., Heckman, T. M., Tremonti, C., et al. 2003, MNRAS, 346, 1055

Kennicutt, R. C., Jr. 1998, ApJ, 498, 541

Kewley, L. J., Dopita, M. A., Sutherland, R. S., Heisler, C. A., \& Trevena, J. 2001, ApJ, 556, 121

Kormendy, J., \& Ho, L. C. 2013, ARA\&A, 51, 511

Larkin, J., Barczys, M., Krabbe, A., et al. 2006, Proc. SPIE, 6269, 62691A

Lehnert, M. D., van Breugel, W. J. M., Heckman, T. M., \& Miley, G. K. 1999, ApJS, 124, 11

Liu, G., Zakamska, N. L., Greene, J. E., Nesvadba, N. P. H., \& Liu, X. 2013a, MNRAS, 436, 2576

Liu, X., Civano, F., Shen, Y., et al. 2013b, ApJ, 762, 110

Luridiana, V., Morisset, C., \& Shaw, R. A. 2015, A\&A, 573, A42

Lyke, J., Do, T., Boehle, A., et al. 2017, Astrophysics Source Code Library, ascl: 1710.021

Madau, P., \& Dickinson, M. 2014, ARA\&A, 52, 415

Magorrian, J., Tremaine, S., Richstone, D., et al. 1998, AJ, 115, 2285

Mantovani, F., Rossetti, A., Junor, W., Saikia, D. J., \& Salter, C. J. 2013, A\&A, 555, A4

McConnell, N. J., \& Ma, C.-P. 2013, ApJ, 764, 184

McLure, R. J., \& Dunlop, J. S. 2004, MNRAS, 352, 1390

McMullin, J. P., Waters, B., Schiebel, D., Young, W., \& Golap, K. 2007, in ASP Conf. Ser. 376, Astronomical Data Analysis Software and Systems XVI, ed. R. A. Shaw, F. Hill, \& D. J. Bell (San Francisco, CA: ASP), 127

Medling, A. M., U, V., Max, C. E., et al. 2015, ApJ, 803, 61

Mieda, E., Wright, S. A., Larkin, J. E., et al. 2014, PASP, 126, 250

Murray, N., Chiang, J., Grossman, S. A., \& Voit, G. M. 1995, ApJ, 451, 498

Murray, N., Quataert, E., \& Thompson, T. A. 2005, ApJ, 618, 569

Nesvadba, N. P. H., Lehnert, M. D., De Breuck, C., Gilbert, A. M., \& van Breugel, W. 2008, A\&A, 491, 407

Osterbrock, D. E., \& Ferland, G. J. 2006, Astrophysics of Gaseous Nebulae and Active Galactic Nuclei (Mill Valley, CA: Univ. Science Books)

Planck Collaboration, Ade, P. A. R., Aghanim, N., et al. 2014, A\&A, 571, A16

Podigachoski, P., Barthel, P. D., Haas, M., et al. 2015, A\&A, 575, A80

Runnoe, J. C., Brotherton, M. S., \& Shang, Z. 2012, MNRAS, 422, 478

Rupke, D. S. N., \& Veilleux, S. 2011, ApJL, 729, L27

Sachdeva, S., Saha, K., \& Singh, H. P. 2017, ApJ, 840, 79

Sánchez, S. F., Jahnke, K., Wisotzki, L., et al. 2004, ApJ, 614, 586

Sanders, D. B., Soifer, B. T., Elias, J. H., et al. 1988, ApJ, 325, 74

Sani, E., Marconi, A., Hunt, L. K., \& Risaliti, G. 2011, MNRAS, 413, 1479

Shen, Y., Richards, G. T., Strauss, M. A., et al. 2011, ApJS, 194, 45

Steinbring, E. 2011, AJ, 142, 172

Stockman, H. S., Moore, R. L., \& Angel, J. R. P. 1984, ApJ, 279, 485

Stone, M., Veilleux, S., Meléndez, M., et al. 2016, ApJ, 826, 111

Storey, P. J., \& Zeippen, C. J. 2000, MNRAS, 312, 813

Sun, A.-L., Greene, J. E., Zakamska, N. L., \& Nesvadba, N. P. H. 2014, ApJ, 790,160 
Treister, E., Schawinski, K., Urry, C. M., \& Simmons, B. D. 2012, ApJL, 758, L39

Vayner, A., Wright, S. A., Do, T., et al. 2016, ApJ, 821, 64

Veilleux, S., Bolatto, A., Tombesi, F., et al. 2017, ApJ, 843, 18

Venemans, B. P., Walter, F., Zschaechner, L., et al. 2016, ApJ, 816, 37

Vestergaard, M., \& Osmer, P. S. 2009, ApJ, 699, 800
Wang, R., Wagg, J., Carilli, C. L., et al. 2013, ApJ, 773, 44

Willott, C. J., Bergeron, J., \& Omont, A. 2015, ApJ, 801, 123

Wong, O. I., Schawinski, K., Kaviraj, S., et al. 2012, MNRAS, 420, 1684

Zubovas, K., \& King, A. 2012, ApJL, 745, L34

Zubovas, K., \& King, A. R. 2014, MNRAS, 439, 400 